\title{
A characterization of Prym varieties
}

\author{
I.Krichever *
}

April 15, 2006

\begin{abstract}
We prove that Prym varieties of algebraic curves with two smooth fixed points of involution are exactly the indecomposable principally polarized abelian varieties whose theta-functions provide explicit formulae for integrable $2 D$ Schrödinger equation.
\end{abstract}

\section{Introduction}

The problem of the characterization of the Prymians among principally polarized abelian varieties is almost as old as the famous Riemann-Schottky problem on the characterization of the Jacobian locus. Until now despite all the efforts it has remained unsolved. Analogs of quite a few geometrical characterizations of the Jacobians for the case of Prym varieties are either unproved or known to be invalid (see reviews [1, 2] and references therein).

The first effective solution of the Riemann-Schottky problem was obtained by T.Shiota ([3]), who proved Novikov's conjecture: the Jacobains of curves are exactly the indecomposable principally polarized abelian varieties whose theta-functions provide explicit solutions of the KP equation. Attempts to prove the analog of Novikov's conjecture for the case of Prym varieties were made in [4, 5, 6]. In [4] it was shown that Novikov-Veselov (NV) equation provides local solution of the characterization problem. In [5, 6] the characterizations of the Prym varieties in terms of BKP and NV equations were proved only under certain additional assumptions. Note, that in [6] a counter example showing that BKP equation has theta-functional solutions which do not correspond to the Prym varieties was constructed.

The goal of this work is to solve the characterization problem of the Prym varieties using the new approach proposed in the author's previous work [7], where it was shown that KP equation contains excessive information and the Jacobian locus can be characterized in terms only of one of its auxiliary linear equations.

\footnotetext{
${ }^{*}$ Columbia University, New York, USA and Landau Institute for Theoretical Physics, Moscow, Russia; e-mail: krichev@math.columbia.edu. Research is supported in part by National Science Foundation under the grant DMS-04-05519.

${ }^{\dagger}$ the revised version
} 
Theorem 1.1 (77) An indecomposable symmetric matrix B with positive definite imaginary part is the matrix of the b-periods of normalized holomorphic differentials on a curve of genus $g$ if and only if there exist $g$-dimensional vectors $U \neq 0, V, A$ such that the equation

$$
\left(\partial_{y}-\partial_{x}^{2}+u\right) \psi=0
$$

is satisfied with

$$
u=-2 \partial_{x}^{2} \ln \theta(U x+V y+Z) \text { and } \psi=\frac{\theta(A+U x+V y+Z)}{\theta(U x+V y+Z)} e^{p x+E y},
$$

where $p, E$ are constants.

Here $\theta(z)=\theta(z \mid B), z=\left(z_{1}, z_{2}, \ldots, z_{g}\right)$ is the Riemann theta-function, defined by the formula

$$
\theta(z)=\sum_{m \in \mathbb{Z}^{g}} e^{2 \pi i(z, m)+\pi i(B m, m)}, \quad(z, m)=m_{1} z_{1}+\cdots+m_{g} z_{g}
$$

The addition formula for the Riemann theta-function directly implies that equation (1.1) with $u$ and $\psi$ as in (1.2) is in fact equivalent to the system of equations

$$
\partial_{V} \Theta[\varepsilon, 0](A / 2)-\partial_{U}^{2} \Theta[\varepsilon, 0](A / 2)-2 p \partial_{U} \Theta[\varepsilon, 0](A / 2)+\left(E-p^{2}\right) \Theta[\varepsilon, 0](A / 2)=0,
$$

where $\Theta[\varepsilon, 0](z)=\theta[\varepsilon, 0](2 z \mid 2 B)$ are level two theta-functions with half-integer characteristics $\varepsilon \in \frac{1}{2} Z_{2}^{g}$.

The characterization of the Jacobian locus given by Theorem 1.1 is stronger than that given in terms of the KP equation (see details in [8], where Theorem 1.1 was proved under the assumption that the closure $\langle A\rangle$ of the subgroup of $X$ generated by $A$ is irreducible). In terms of the Kummer map,

$$
\kappa: Z \in X \longmapsto\left\{\Theta\left[\varepsilon_{1}, 0\right](Z): \cdots: \Theta\left[\varepsilon_{2^{g}}, 0\right](Z)\right\} \in \mathbb{C P}^{2^{g}-1},
$$

the statement of Theorem 1.1 is equivalent to the characterization of the Jacobians via flexes of the Kummer varieties, which is a particular case of the trisecant conjecture, first formulated in 9 .

The Prym variety of a smooth algebraic curve $\Gamma$ with involution $\sigma: \Gamma \longmapsto \Gamma$ is defined as the odd subspace $\mathcal{P}(\Gamma) \subset J(\Gamma)$ of the Jacobian with respect to the involution $\sigma^{*}: J(\Gamma) \longmapsto$ $J(\Gamma)$ induced by $\sigma$. It is principally polarized only if $\sigma$ has no fixed points or has two fixed points $P_{ \pm}$. In this work we consider only the second case.

Let $\Gamma$ be a smooth algebraic curve with involution $\sigma$ having two fixed points $P_{ \pm}$. From the Riemann-Hurwitz formula it follows that if the genus of the factor-curve $\Gamma_{0}=\Gamma / \sigma$ equals $g$, then the genus $\Gamma$ is equal to $2 g$. It is known that on $\Gamma$ there exists a basis of cycles $a_{i}, b_{i}$ with the canonical matrix of intersections $a_{i} \cdot a_{j}=b_{i} \cdot b_{j}=0, a_{i} \cdot b_{j}=\delta_{i j}, \quad 1 \leq i, j \leq 2 g$, such that $\sigma\left(a_{k}\right)=-a_{g+k}, \sigma\left(b_{k}\right)=-b_{g+k}, 1 \leq k \leq g$. If $d \omega_{i}$ are normalized holomorphic differentials on $\Gamma$, then the differential $d u_{k}=d \omega_{k}+d \omega_{g+k}$ are odd $\sigma^{*}\left(d u_{k}\right)=-d u_{k}$. By definition they are called the normalized holomorphic Prym differentials. The matrix of their $b$-periods

$$
\Pi_{k j}=\oint_{b_{k}} d u_{j}, \quad 1 \leq k, j \leq g
$$


is symmetric, has positive definite imaginary part, and defines the Prym theta-function $\theta_{P r}=\theta(z \mid \Pi)$.

Before presenting our main result it is necessary to mention that the Prym variety remains non-degenerate (compact) under certain degenerations of the curve. No characterization of Prym varieties given in terms of equations for the matrix $\Pi$ of periods of the Prym differentials can single out the possibility of such degenerations. An algebraic curve $\Gamma$ that is smooth outside fixed points $P_{+}, P_{-}, Q_{1}, Q_{2}, \ldots, Q_{k}$ of its involution $\sigma$, where $P_{ \pm}$are smooth and $Q_{k}$ are simple double points at which $\sigma$ does not permute branches of $\Gamma$, will be denoted below by $\left\{\Gamma, \sigma, P_{ \pm}, Q_{k}\right\}$.

Theorem 1.2 An indecomposable principally polarized abelian variety $(X, \theta)$ is the Prym variety of a curve of type $\left\{\Gamma, \sigma, P_{ \pm}, Q_{k}\right\}$, if and only if there exist $g$-dimensional vectors $U \neq 0, V \neq 0, A$ such that one of the following equivalent conditions holds:

(A) The equation

$$
\left(\partial_{x} \partial_{t}+u\right) \psi=0
$$

is satisfied with

$$
u=2 \partial_{x t}^{2} \ln \theta(U x+V t+Z)+C, \quad \text { and } \quad \psi=\frac{\theta(A+U x+V t+Z)}{\theta(U x+V t+Z)} e^{p x+E t},
$$

where $C, p, E$ are constants.

(B) The equations

$$
\partial_{U V}^{2} \Theta[\varepsilon, 0](A / 2)+p \partial_{V} \Theta[\varepsilon, 0](A / 2)+E \partial_{U} \Theta[\varepsilon, 0](A / 2)+C \Theta[\varepsilon, 0](A / 2)=0
$$

are satisfied for all $\varepsilon \in \frac{1}{2} Z_{2}^{g}$.

(C) The equation

$$
\partial_{U} \theta \partial_{V} \theta\left(\partial_{U}^{2} \partial_{V}^{2} \theta\right)+\partial_{U}^{2} \theta \partial_{V}^{2} \theta\left(\partial_{U} \partial_{V} \theta\right)-\partial_{U}^{2} \theta \partial_{V} \theta\left(\partial_{U} \partial_{V}^{2} \theta\right)-\left.\partial_{U} \theta \partial_{V}^{2} \theta\left(\partial_{U}^{2} \partial_{V} \theta\right)\right|_{\Theta}=0
$$

is valid on the theta-divisor $\{Z \in \Theta: \theta(Z)=0\}$.

The equivalence of $(A)$ and $(B)$ is a direct corollary of the addition formula for the thetafunction. The "if" part of $(A)$ follows from the construction of integrable $2 D$ Schrödinger operators given in [10]. This construction is presented in the next section.

The statement $(C)$ is actually what we use for the proof of the theorem. It is stronger than $(A)$. The implication $(A) \rightarrow(C)$ does not require the explicit theta-functional form of $\psi$. It is enough to require only that equation (1.7) with $u$ as in (1.8) has local meromorphic in $x$ (or $t$ ) solutions which are holomorphic outside the divisor $\theta(U x+V t+Z)=0$.

To put it more precisely, let us consider a function $\tau(x, t)$ which is a holomorphic function of $x$ in some domain where the equation $\tau(x, t)=0$ has a simple root $\eta(t)$. It turns out that 
equation (1.7) with the potential $u=2 \partial_{x t}^{2} \ln \tau+C$, where $C$ is a constant, has a meromorphic solution in $D$, if this root satisfies the equation

$$
\ddot{\eta} v-\dot{\eta} \dot{v}+2 \dot{\eta}^{2} w=0
$$

where $v=v(t), w=w(t)$ are the first coefficients of the Laurent expansion of $u$ at $\eta$

$$
u(x, t)=\frac{2 \dot{\eta}}{(x-\eta)^{2}}+v+w(x-\eta)+\cdots
$$

and "dots" stand for $t$-derivatives. Straightforward but tedious computations with expansion of $\theta$ at the generic points of its divisor $\Theta$ show that equation (1.11) in the case when $\tau=$ $\theta(U x+V t+Z)$ is equivalent to equation (1.10).

Note that equations (1.11) are analogues of the equations derived in [8] and called in [7] the formal Calogero-Moser system. In a similar way, if we represent an entire function $\tau$ as a product

$$
\tau(x, t)=c(t) \prod_{i}\left(x-x_{i}(t)\right)
$$

then equation (1.11) takes the form

$$
\sum_{j \neq i}\left[\frac{\ddot{x}_{i} \dot{x}_{j}-\dot{x}_{i} \ddot{x}_{j}}{\left(x_{i}-x_{j}\right)^{2}}-\frac{2 \dot{x}_{i} \dot{x}_{j}\left(\dot{x}_{i}+\dot{x}_{j}\right)}{\left(x_{i}-x_{j}\right)^{3}}\right]=0 .
$$

At the moment the only reason for presenting equations (1.14) is to show that in the case when $\tau$ is a rational, trigonometric or elliptic polynomial the system (1.11) gives well-defined equations of motion for a multi-particle system. ${ }^{1}$

At the beginning of section 3 we derive equation (1.11) and show that equation (1.10) is sufficient for the local existence of wave solutions of (1.7) having the form

$$
\psi_{ \pm}(x, t, k)=e^{k t_{ \pm}}\left(1+\sum_{s=1}^{\infty} \xi_{s}^{ \pm}(x, t) k^{-s}\right), t_{+}=x, t_{-}=t
$$

and such that

$$
\xi_{s}^{ \pm}=\frac{\tau_{s}^{ \pm}\left(U x+V t+Z, t_{\mp}\right)}{\theta(U x+V t+Z)}, Z \notin \Sigma_{ \pm},
$$

where $\tau_{s}^{ \pm}\left(Z, t_{\mp}\right)$, as a function of $Z$, is holomorphic in some open domain in $\mathbb{C}^{g}$. Here and below $\Sigma_{ \pm} \subset \Theta$ are subsets of the theta-divisor invariant under the shifts along constant vector fields $\partial_{U}$ or $\partial_{V}$, respectively.

The coefficients $\xi_{s}^{ \pm}$of the wave solutions are defined recurrently by the equations $\partial_{\mp} \xi_{s+1}^{ \pm}=$ $-\partial_{x t} \xi_{s}-u \xi_{s}$. The local existence of meromorphic solutions requires vanishing of the residues of the nonhomogeneous terms. That is controlled by equation (1.11). At the local level the main problem is to find the translational invariant normalization of $\xi_{s}^{ \pm}$which defines wave solutions uniquely up to a $(x, t)$-independent factor.

\footnotetext{
${ }^{1}$ A.Zotov noticed that equation (1.14) is equivalent to the equations $\ddot{x}_{i}=2 \sum_{j \neq i} \dot{x}_{i} \dot{x}_{j} /\left(x_{i}-x_{j}\right)$ and, therefore, can be regarded as a limiting case of the Ruijesenaars-Schneider system.
} 
Following the ideas of [11] and [7] we fix such a normalization using extensions of $\xi_{s}^{ \pm}$along the affine subspaces $Z+\mathbb{C}_{ \pm}^{d}$, where $\mathbb{C}_{ \pm}^{d}$ are universal covers of the abelian subvarieties $Y_{ \pm} \subset X$ which are closures of the subgroups $U x$ and $V t$ in $X$, respectively. The corresponding wave solutions are called $\lambda$-periodic.

In the last section we show that for each $Z \notin \Sigma_{ \pm}$a local $\lambda$-periodic wave solution is the common eigenfunction of a commutative ring $\mathcal{A}_{ \pm}^{Z}$ of ordinary differential operators. The coefficients of these operators are independent of ambiguities in the construction of $\psi$. The theory of commuting differential operators [12, 13, 14, 15] implies then that the correspondence $Z \longmapsto \mathcal{A}_{ \pm}^{Z}$ defines a map $j$ of $X \backslash \Sigma_{ \pm}$into the space $\overline{\operatorname{Pic}(\Gamma)}$ of torsion-free rang 1 sheafs $\mathcal{F}$ on $Z$-independent spectral curve of $\mathcal{A}^{Z}$. That allows us to make the next crucial step and prove the global existence of the wave function. The global existence of the wave function implies that for the generic $Z \notin \Sigma_{ \pm}$the orbit of $\mathcal{A}^{Z}$ under the NV flows defines an imbedding $i_{Z}$ of the Prym variety $\mathcal{P}(\Gamma)$ of the spectral curve into $X$. Therefore, the Prym variety is compact. That implies the explicit description of possible types of singular points of $\Gamma$. The final step in the proof of the main theorem is to show that there are not singular points of the multiplicity bigger then 2 .

\section{Integrable 2D Schrödinger operators}

In this section we present necessary facts from the theory of integrable $2 D$ Schrödinger equations and related hierarchies.

Let $\Gamma$ be a smooth algebraic curve of genus $g$ with fixed local coordinates $k_{ \pm}^{-1}$ at punctures $P_{ \pm}$and let $t^{( \pm)}=\left\{t_{i}^{( \pm)}\right\}$be finite sets of complex variables. Then according to the general construction of the multi-point Baker-Akhiezer functions ([12, 13]) for each non-special effective divisor $D=\left\{\gamma_{1}, \ldots, \gamma_{g}\right\}$ of degree $g$ there exists a unique function $\psi_{0}\left(t^{(+)}, t^{(-)}, P\right)$, which, as a function of the variable $P \in \Gamma$, is meromorphic on $\Gamma \backslash P_{ \pm}$, where it has poles at $\gamma_{s}$ of degree not greater then the multiplicity of $\gamma_{s}$ in $D$. In the neighborhood of $P_{ \pm}$the function $\psi_{0}$ has the form

$$
\psi_{0}=e^{\sum_{i} k^{i} t_{i}^{( \pm)}}\left(\sum_{s=0}^{\infty} \xi_{s}^{ \pm}(t) k^{-s}\right), \quad \xi_{0}^{+}=1,
$$

where $k=k_{ \pm}^{-1}(P)$ and $t=\left\{t^{(+)}, t^{(-)}\right\}$.

The uniqueness of $\psi_{0}$ implies that for each positive integer $n$ there exists unique differential operators $B_{n}^{ \pm}$in the variables $t_{1}^{ \pm}$

$$
B_{n}^{ \pm}=\partial_{ \pm}^{n}+\sum_{i=0}^{n-1} v_{n, i}^{ \pm}(t) \partial_{ \pm}^{n-i}, \quad \partial_{ \pm}=\partial / \partial t_{1}^{ \pm}
$$

such that

$$
\left(\partial_{t_{n}^{ \pm}}-B_{n}^{ \pm}\right) \psi_{0}=0
$$

Equations (2.3) directly imply

$$
\left[\partial_{t_{n}^{ \pm}}-B_{n}^{ \pm}, \partial_{t_{m}^{ \pm}}-B_{m}^{ \pm}\right]=0 .
$$


In other words, the operators $B_{n}^{ \pm}$satisfy zero-curvature equations which define two copies of the KP hierarchy with respect to the times $t_{n}^{ \pm}$.

The two-point Baker-Akhiezer function with separated variables was introduced in [16] where it was proved that in addition to (2.3) it satisfies the equation

$$
H \psi_{0}=\left(\partial_{+} \partial_{-}+w \partial_{+}+u\right) \psi_{0}=0
$$

where

$$
w=-\partial_{-} \ln \xi_{0}^{-}, \quad u=-\partial_{+} \partial_{-} \ln \xi_{1}^{+} .
$$

The operator $H$ defined in the left hand side of (2.5) "couples" two copies of the KP hierarchy corresponding to the punctures $P_{ \pm}$via the equation

$$
\left[\partial_{t_{n}^{+}}-B_{n}^{+}, \partial_{t_{m}^{-}}-B_{m}^{-}\right]=D_{n m} H .
$$

The sense of (2.7) is as follows. Each differential operator $\mathcal{D}$ in the two variables $t_{1}^{ \pm}$can be uniquely represented in the form

$$
\mathcal{D}=D H+D^{+}+D^{-},
$$

where $D^{ \pm}$are ordinary differential operators in the variables $t_{1}^{ \pm}$, respectively. The equation (2.7) is just the statement that the second and the third terms in the corresponding representation of the left hand side of (2.7) are equal to zero. This implies $n+m-1$ equations on $n+m-1$ unknown functions (the coefficients of $B_{n}^{+}$and $B_{m}^{-}$). Therefore, the operator equation (2.8), is equivalent to the well-defined system of non-linear partial differential equations.

Explicit theta-functional formulae for the solutions of these equations follow from the theta-functional formula for the Baker-Akhiezer function

$$
\psi_{0}=\frac{\theta\left(A(P)+\sum_{i}\left(U_{i}^{+} t_{i}^{+}+U_{i}^{-} t_{i}^{-}\right)+Z\right) \theta\left(A\left(P_{+}\right)+Z\right)}{\theta\left(A\left(P_{+}\right)+\sum_{i}\left(U_{i}^{+} t_{i}^{+}+U_{i}^{-} t_{i}^{-}\right)+Z\right) \theta(A(P)+Z)} e^{\sum_{i}\left(t_{i}^{+} \Omega_{i}^{+}(P)+t_{i}^{-} \Omega_{i}^{-}(P)\right)}
$$

Here:

a) $\theta(z)=\theta(z \mid B)$ is the Riemann theta-function defined by the matrix $B$ of $b$-periods of normalized holomorphic differentials $d \omega_{k}$ on $\Gamma$.

b) $\Omega_{i}^{ \pm}(P)=\int^{P} d \Omega_{i}^{ \pm}$is the Abelian integral corresponding to the normalized, $\oint_{a_{k}} d \Omega_{i}^{ \pm}=0$, meromorphic differential on $\Gamma$ with the only pole of the form

$$
d \Omega_{i}^{ \pm}=d k_{ \pm}^{i}\left(1+O\left(k_{ \pm}^{-i-1}\right)\right)
$$

at the puncture $P_{ \pm}$;

c) $2 \pi i U_{j}^{ \pm}$is a vector of $b$-periods of the differential $d \Omega_{j}^{ \pm}$with the coordinates

$$
U_{j, k}^{ \pm}=\frac{1}{2 \pi i} \oint_{b_{k}} d \Omega_{j}^{ \pm}
$$

d) $A(P)$ is the Abel transform, i.e. it is a vector with the coordinates $A_{k}(P)=\int^{P} d \omega_{k}$; 
e) $Z$ is an arbitrary vector (it corresponds to the divisor of poles of Baker-Akhiezer function).

Taking the evaluation at $P_{-}$and the expansion at $P_{+}$of the regular factor in (2.9), one gets theta-functional formulae for the coefficients (2.6) of the corresponding 2D Schrödinger operator:

$$
\begin{aligned}
& w=-\partial_{-} \ln \left(\frac{\theta\left(A\left(P_{-}\right)+\sum_{i}\left(U_{i}^{+} t_{i}^{+}+U_{i}^{-} t_{i}^{-}\right)+Z\right)}{\theta\left(A\left(P_{+}\right)+\sum_{i}\left(U_{i}^{+} t_{i}^{+}+U_{i}^{-} t_{i}^{-}\right)+Z\right)}\right), \\
& u=\partial_{+} \partial_{-} \ln \left(\theta\left(A\left(P_{+}\right)+\sum_{i}\left(U_{i}^{+} t_{i}^{+}+U_{i}^{-} t_{i}^{-}\right)+Z\right)\right)+C,
\end{aligned}
$$

where the constant $C$ is equal to $C=\operatorname{res}_{P_{+}} \Omega_{-} d \Omega_{+}$. Note, that the second factors in the numerator and denominator of the formula (2.9) are $t$-independent. Therefore, the function $\psi$ given by the following formula

$$
\psi=\frac{\theta\left(A(P)+\sum_{i}\left(U_{i}^{+} t_{i}^{+}+U_{i}^{-} t_{i}^{-}\right)+Z\right)}{\theta\left(A\left(P_{+}\right)+\sum_{i}\left(U_{i}^{+} t_{i}^{+}+U_{i}^{-} t_{i}^{-}\right)+Z\right)} e^{\sum_{i}\left(t_{i}^{+} \Omega_{i}^{+}(P)+t_{i}^{-} \Omega_{i}^{-}(P)\right)}
$$

is a solution of the same linear equations as $\psi_{0}$. Below $\psi$ given by (2.14) will be called non-normalized Baker-Akhiezer function.

Potential operators. From now on we will consider only potential Schrödinger operators $H=\partial_{+} \partial_{-}+u$. The reduction of the above described algebraic-geometrical construction to the potential case was found in [10]. The corresponding algebraic-geometrical data are singled out by the following constraints:

(i) The curve $\Gamma$ should be a curve with involution $\sigma: \Gamma \rightarrow \Gamma$ which has two fixed points $P_{ \pm}$

(ii) The equivalence class $[D] \in J(\Gamma)$ of the divisor $D$ should satisfy the equation

$$
[D]+[\sigma(D)]=K+P_{+}+P_{-} \in J(\Gamma),
$$

where $K$ is the canonical class, i.e. the equivalence class of the zero-divisor of a holomorphic differential on $\Gamma$.

Equation (2.15) is equivalent to the condition that the divisor $D+\sigma(D)$ is the zero divisor of a meromorphic differential $d \Omega$ on $\Gamma$ with simple poles at the punctures $P_{ \pm}$. The differential $d \Omega$ is even with respect to the involution and descends to a meromorphic differential on the factor-curve $\Gamma_{0}=\Gamma / \sigma$. The projection $\pi: \Gamma \longmapsto \Gamma_{0}=\Gamma / \sigma$ represents $\Gamma$ as a two-sheet covering of $\Gamma_{0}$ with 2 branch points $P_{ \pm}$. In this realization the involution $\sigma$ is a permutation of the sheets. For $P \in \Gamma$ we denote the point $\sigma(P)$ by $P^{\sigma}$. From the Riemann-Hurwitz formula it follows that the genus $g$ of $\Gamma$ equals $g=2 g_{0}$, where $g_{0}$ is the genus of $\Gamma_{0}$. Note that the divisors that satisfy (2.15) are parameterized by the points $Z_{0}$ of the Prym variety $\mathcal{P}(\Gamma) \subset J(\Gamma)$.

Theorem 2.1 ([10]). Let a smooth algebraic curve $\Gamma$ and an effective divisor $D$ satisfy the constraints $(i),(i i)$. Let $k_{ \pm}^{-1}(P)$ be odd local coordinates in the neighborhoods of the fixed 
points $P_{ \pm}$, i.e. $k_{ \pm}(P)=-k_{ \pm}(\sigma(P))$, and let all the even times vanish, i.e. $t_{2 i}^{ \pm}=0$. Then the corresponding $2 D$ Schrödinger operator is potential, i.e. $w=0$.

In [10]) it was also found that for the potential operators the formulae (2.9) and (2.13) can be expressed in terms of the Prym theta-function. For further use it is enough to present these formulae for the case of only two nontrivial variables $x=t_{1}^{+}, t=t_{1}^{-}$:

$$
\begin{gathered}
\psi=\frac{\theta_{P r}\left(A^{\operatorname{Pr}}(P)+U x+V t+Z\right)}{\theta_{P r}\left(A^{\operatorname{Pr}}\left(P_{+}\right)+U x+V t+Z\right)} e^{x \Omega_{1}^{+}+t \Omega_{1}^{-}} \\
u=2 \partial_{+} \partial_{-} \ln \theta_{P r}\left(A^{\operatorname{Pr}}\left(P_{+}\right)+x U+t V+Z\right)+C .
\end{gathered}
$$

Here $A^{P r}: \Gamma \longmapsto \mathcal{P}(\Gamma)$ is the Abel-Prym map defined by the Prym differentials, i.e. $A^{\operatorname{Pr}}(P)$ is a vector with the coordinates $A_{k}^{P r}(P)=\int^{P} d u_{k}$.

In 17, 18] it was proved that for the case of smooth periodic potentials $u(x, t)$ (considered as a function of real variables $x, t)$ the conditions found by Novikov and Veselov are sufficient and necessary.

\section{$3 \quad \lambda$-periodic wave solutions}

To begin with, let us show that equations (1.11) are the necessary condition of the existence of a meromorphic solution to equation (1.7).

Let $\tau(x, t)$ be a smooth $t$-parametric family of holomorphic functions of the variable $x$ in some open domain $D \subset \mathbb{C}$. Suppose that in $D$ the function $\tau$ has a simple zero,

$$
\tau(\eta(t), t)=0, \tau_{x}(\eta(t), t) \neq 0 .
$$

Lemma 3.1 If equation (1.7) with the potential $u=2 \partial_{x t}^{2} \ln \tau(x, t)+C$, where $C$ is a constant, has a meromorphic solution $\psi_{0}(x, t)$, then equation (1.11) holds.

Proof. Consider the Laurent expansions of $\psi_{0}$ and $u$ in the neighborhood of $\eta$ :

$$
\begin{gathered}
u=\frac{2 \dot{\eta}}{(x-\eta)^{2}}+v+w(x-\eta)+\ldots \\
\psi_{0}=\frac{\alpha}{x-\eta}+\beta+\gamma(x-\eta)+\ldots
\end{gathered}
$$

(All the coefficients in these expansions are smooth functions of the variable $t$ ). Substitution of (3.23.3) into (1.7) gives a system of equations. The first three of them are

$$
\begin{gathered}
\dot{\alpha}-2 \dot{\eta} \beta=0, \\
2 \dot{\eta} \gamma+\alpha v=0,
\end{gathered}
$$




$$
\dot{\gamma}+v \beta+\alpha w=0 .
$$

Taking the $t$-derivative of the second equation and using two others we get (1.11).

Let us show that equations (1.11) are sufficient for the existence of meromorphic wave solutions.

Lemma 3.2 Suppose that equation (1.11) for the zero of $\tau(x, t)$ holds. Then equation (1.7) has wave solutions of the form

$$
\psi=e^{k t}\left(1+\sum_{s=1}^{\infty} \xi_{s}(x, t) k^{-s}\right)
$$

such that the coefficients $\xi_{s}$ have simple poles at $\eta$ and are holomorphic everywhere else in D.

Proof. Substitution of (3.7) into (1.7) gives a recurrent system of equations

$$
\xi_{s+1}^{\prime}=-\partial_{x t}^{2} \xi_{s}-u \xi_{s}
$$

We are going to prove by induction that this system has meromorphic solutions with simple poles at $\eta$.

Let us expand $\xi_{s}$ at $\eta$ :

$$
\xi_{s}=\frac{r_{s}}{x-\eta}+r_{s 0}+r_{s 1}(x-\eta)+\cdots
$$

Suppose that $\xi_{s}$ is defined, and equation (3.8) has a meromorphic solution. Then the right hand side of (3.8) has the zero residue at $x=\eta$, i.e.,

$$
\operatorname{res}_{\eta}\left(\partial_{x t}^{2} \xi_{s}+u \xi_{s}\right)=v r_{s}+2 \dot{\eta} r_{s 1}=0
$$

We need to show that the residue of the next equation also vanishes. From (3.8) it follows that the coefficients of the Laurent expansion for $\xi_{s+1}$ are equal to

$$
\begin{gathered}
r_{s+1}=-\dot{r}_{s}+2 \dot{\eta} r_{s 0}, \\
r_{s+1,1}=-v r_{s 0}-w r_{s}-\dot{r}_{s 1} .
\end{gathered}
$$

These equations and equation (3.10) imply

$$
-\left(v r_{s+1}+2 \dot{\eta} r_{s+1,1}\right)=2 \dot{\eta} w r_{s}+v \dot{r}_{s}+2 \dot{\eta} \dot{r}_{s, 1}=\partial_{t}\left(v r_{s}+2 \dot{\eta} r_{s, 1}\right)-\left(\dot{v}-\frac{\ddot{\eta}}{\dot{\eta}} v-2 \dot{\eta} w\right) r_{s}=0
$$

and the lemma is proved.

Our next goal is to fix a translation-invariant normalization of $\xi_{s}$ which defines wave functions uniquely up to a $(x, t)$-independent factor. It is instructive to consider first the case of the periodic potentials $u(x+1, t)=u(x, t)$ (compare with [1]]). 
Equations (3.8) are solved recursively by the formulae

$$
\begin{aligned}
& \xi_{s+1}(x, t)=c_{s+1}(t)+\xi_{s+1}^{0}(x, t), \\
& \xi_{s+1}^{0}(x, t)=-\partial_{t} \xi_{s}-\int_{x_{0}}^{x} u \xi_{s} d x,
\end{aligned}
$$

where $c_{s}(t)$ are arbitrary functions of the variable $t$. Let us show that the periodicity condition $\xi_{s}(x+1, t)=\xi_{s}(x, t)$ defines these functions uniquely up to constants. Assume that $\xi_{s-1}$ is known and satisfies the condition that the corresponding function $\xi_{s}^{0}$ is periodic. The choice of the function $c_{s}(t)$ does not affect the periodicity property of $\xi_{s}$, but it does affect the periodicity in $x$ of the function $\xi_{s+1}^{0}(x, t)$. In order to make $\xi_{s+1}^{0}(x, t)$ periodic, the function $c_{s}(t)$ should satisfy the linear differential equation

$$
\partial_{t} c_{s}(t)+\int_{x_{0}}^{x_{0}+1} u(x, t)\left(c_{s}(t)+\xi_{s}^{0}(x, t)\right) d x=0 .
$$

This defines $c_{s}$ uniquely up to a constant.

In the general case, when $u$ given by (1.8) is quasi-periodic, the normalization of the wave functions is defined along the same lines.

Let $\Theta_{1}$ be defined by the equations $\Theta_{1}=\left\{Z: \theta(Z)=\partial_{U} \theta(Z)=0\right\}$, where $\partial_{U}$ is a constant vector-field on $\mathbb{C}^{g}$, corresponding to the vector $U$ in (1.8). The $\partial_{U}$-invariant subset $\Sigma$ of $\Theta_{1}$ will be called the singular locus.

Consider the closure $Y_{U}=\langle U x\rangle$ of the group $U x$ in $X$. Shifting $Y_{U}$ if needed, we may assume, without loss of generality, that $Y_{U}$ is not in the singular locus, $Y_{U} \notin \Sigma$. Then, for a sufficiently small $t$, we have $Y_{U}+V t \notin \Sigma$ as well. Consider the restriction of the thetafunction onto the affine subspace $\mathbb{C}^{d}+V t$, where $\mathbb{C}^{d}=\pi^{-1}\left(Y_{U}\right)$, and $\pi: \mathbb{C}^{g} \rightarrow X=\mathbb{C}^{g} / \Lambda$ is the universal cover of $X$ :

$$
\tau(z, t)=\theta(z+V t), \quad z \in \mathbb{C}^{d} .
$$

The function $u(z, t)=2 \partial_{U} \partial_{t} \ln \tau+C$ is periodic with respect to the lattice $\Lambda_{U}=\Lambda \cap \mathbb{C}^{d}$ and, for fixed $t$, has a double pole along the divisor $\Theta^{U}(t)=(\Theta-V t) \cap \mathbb{C}^{d}$.

Lemma 3.3 Let equation (1.10) hold and let $\lambda$ be a vector of the sublattice $\Lambda_{U}=\Lambda \cap \mathbb{C}^{d} \subset$ $\mathbb{C}^{g}$. Then:

(i) equation (1.7) with the potential $u(U x+z, t)$ has a wave solution of the form $\psi=$ $e^{k t+b x k^{-1}} \phi(U x+z, t, k)$ such that the coefficients $\xi_{s}(z, t)$ of the formal series

$$
\phi(z, t, k)=1+\sum_{s=1}^{\infty} \tilde{\xi}_{s}(z, t) k^{-s}
$$

are $\lambda$-periodic meromorphic functions of the variable $z \in \mathbb{C}^{d}$ with a simple pole at the divisor $\Theta^{U}(t)$, i.e.

$$
\tilde{\xi}_{s}(z+\lambda, t)=\tilde{\xi}_{s}(z, t)=\frac{\tau_{s}(z, t)}{\tau(z, t)} ;
$$


(ii) $\phi(z, t, k)$ is unique up to a factor $\rho(z, k)$ that is $t$-independent, $\partial_{U}$-invariant and holomorphic in $z$,

$$
\phi_{1}(z, t, k)=\phi(z, t, k) \rho(z, k), \partial_{U} \rho=0
$$

Proof. The functions $\tilde{\xi}_{s}(z)$ are defined recursively by the equations

$$
\partial_{U} \tilde{\xi}_{s+1}=-\partial_{U} \partial_{t} \tilde{\xi}_{s}-(u+b) \tilde{\xi}_{s}-b \partial_{t} \tilde{\xi}_{s-1}
$$

A particular solution of the first equation $\partial_{U} \tilde{\xi}_{1}=-u-b$ is given by the formula

$$
\tilde{\xi}_{1}^{0}=-2 \partial_{t} \ln \tau-(l, z)(b+C),
$$

where $(l, z)$ is a linear form on $\mathbb{C}^{d}$ given by the scalar product of $z$ with a vector $l \in \mathbb{C}^{d}$ such that $(l, U)=1$. By definition, the vector $\lambda$ is in $Y_{U}$. Therefore, $(l, \lambda) \neq 0$. The periodicity condition for $\tilde{\xi}_{1}^{0}$ defines the constant $b$, which depends only on a choice of the lattice vector $\lambda$. From the monodromy properties of $\theta$ it follows that without loss of generality we may assume that $\lambda$ is chosen such that the corresponding constant $b$ is not equal to zero, i.e.

$$
b=-C+(l, \lambda)^{-1}\left(2 \partial_{t} \ln \tau(z, t)-2 \partial_{t} \ln \tau(z+\lambda, t)\right) \neq 0
$$

Note, that the second factor in (3.7) and the series $\phi$ in (3.18) differ by the factor $e^{b x k^{-1}}$, which does not affect the results of the previous lemma. Therefore, equations (1.11) are sufficient for the local solvability of (3.21) in any domain, where $\tau(z+U x, t)$ has simple zeros, i.e. outside the set $\Theta_{1}^{U}(t)=\left(\Theta_{1}-V t\right) \cap \mathbb{C}^{d}$. Recall that $\Theta_{1}=\Theta \cap \partial_{U} \Theta$. This set does not contain a $\partial_{U}$-invariant line because such line is dense in $Y_{U}$. Therefore, the sheaf $\mathcal{V}_{0}$ of $\partial_{U}$-invariant meromorphic functions on $\mathbb{C}^{d} \backslash \Theta_{1}^{U}(t)$ with poles along the divisor $\Theta^{U}(t)$ coincides with the sheaf of holomorphic $\partial_{U}$-invariant functions. That implies the vanishing of $H^{1}\left(C^{d} \backslash \Theta_{1}^{U}(t), \mathcal{V}_{0}\right)$ and the existence of global meromorphic solutions $\xi_{s}^{0}$ of (3.21) which have a simple pole at the divisor $\Theta^{U}(t)$ (see details in [3, 19]).

Let us assume, as in the example above, that a $\lambda$-periodic solution $\tilde{\xi}_{s-1}$ is known and that it satisfies the condition that there exists a $\lambda$-periodic solution $\tilde{\xi}_{s}^{0}$ of the next equation such that the equation

$$
\partial_{U} \chi_{s}=-(u+b) \tilde{\xi}_{s}^{0}-b \partial_{t} \tilde{\xi}_{s-1}
$$

has a $\lambda$-periodic solution. If $\tilde{\xi}_{s}^{0}$ and a particular solution $\chi_{s}^{*}$ of (13.24) are fixed, then $\tilde{\xi}_{s+1}^{*}=$ $\partial_{t} \tilde{\xi}_{s}^{0}+\chi_{s}^{*}$ is a $\lambda$-periodic solution of (3.21) for $\tilde{\xi}_{s}^{0}$.

A choice of a $\lambda$-periodic $\partial_{U}$-invariant function $c_{s}(z, t)$ does not affect the periodicity property of $\tilde{\xi}_{s}=c_{s}+\tilde{\xi}_{s}^{0}$. It changes the right hand side of (3.24). A particular solution of the new equation is given by the formula $\chi_{s}^{0}=\chi_{s}^{*}+c_{s} \tilde{\xi}_{1}^{0}$. Therefore, $\tilde{\xi}_{s+1}^{0}=\partial_{t} \tilde{\xi}_{s}+\chi_{s}^{0}$ is a $\lambda$-periodic solution of (3.21) for $\tilde{\xi}_{s}$. The choice of $c_{s}$ does affect the existence of periodic solutions of the equation

$$
\partial_{U} \chi_{s+1}=-(u+b) \tilde{\xi}_{s+1}^{0}-b \partial_{t} \tilde{\xi}_{s}
$$

Let $\tilde{\chi}_{s+1}$ be a solution of the equation

$$
\partial_{U} \tilde{\chi}_{s+1}=-(u+b) \tilde{\xi}_{s+1}^{*}-b \partial_{t} \tilde{\xi}_{s}^{0} .
$$


Then the function

$$
\chi_{s+1}(z, t)=\tilde{\chi}_{s+1}(z, t)+\frac{1}{2} c_{s}(z, t)\left(\xi_{1}^{0}(z, t)\right)^{2}+\left(\xi_{1}^{0}(z, t)-(l, z) b\right) \partial_{t} c_{s}(z, t),
$$

is a solution of (3.25). In order to make $\chi_{s+1}$ periodic, the function $c_{s}(z, t)$ should satisfy the linear differential equation

$$
\partial_{t} c_{s}(z, t)=((l, \lambda) b)^{-1}\left(\tilde{\chi}_{s+1}(z+\lambda, t)-\tilde{\chi}_{s+1}(z, t)\right)
$$

This equation, together with the initial condition $c_{s}(z)=c_{s}(z, 0)$ uniquely defines $c_{s}(z, t)$. The induction step is then completed. We have shown that the ratio of two periodic formal series $\phi_{1}$ and $\phi$ is $t$-independent. Therefore, equation (3.20), where $\rho(z, k)$ is defined by the evaluation of both the sides at $t=0$, holds. The lemma is thus proven.

Corollary 3.1 Let $\lambda_{1}, \ldots, \lambda_{d}$ be a set of linear independent vectors of the lattice $\Lambda_{U}$ and let $z_{0}$ be a point of $\mathbb{C}^{d}$. Then, under the assumptions of the previous lemma, there is a unique wave solution of equation (1.7) such that the corresponding formal series $\phi\left(z, t, k ; z_{0}\right)$ is quasi-periodic with respect to $\Lambda_{U}$, i.e. for $\lambda \in \Lambda_{U}$

$$
\phi\left(z+\lambda, t, k ; z_{0}\right)=\phi\left(z, t, k ; z_{0}\right) \mu_{\lambda}(k)
$$

and satisfies the normalization conditions

$$
\mu_{\lambda_{i}}(k)=1, \quad \phi\left(z_{0}, 0, k ; z_{0}\right)=1 .
$$

The proof is identical to that in the part (b) of Lemma 12 in [3] (compare with the proof of the corollary in [7]).

\section{The spectral curve}

In this section we show that $\lambda$-periodic wave solutions of equation (1.7), with $u$ as in (1.8), are common eigenfunctions of rings of commuting operators and identify $X$ with the Prym variety of the spectral curve of these rings.

Note that a simple shift $z \rightarrow z+Z$, where $Z \notin \Sigma$, gives $\lambda$-periodic wave solutions with meromorphic coefficients along the affine subspaces $Z+\mathbb{C}^{d}$. These $\lambda$-periodic wave solutions are related to each other by $t$-independent, $\partial_{U}$-invariant factor. Therefore choosing in the neighborhood of any $Z \notin \Sigma$, a hyperplane orthogonal to the vector $U$ and fixing initial data on this hyperplane at $t=0$, we define the corresponding series $\phi(z+Z, t, k)$ as a local meromorphic function of $Z$ and the global meromorphic function of $z$.

Lemma 4.1 Let equation (1.10) hold. Then there is a unique pseudo-differential operator

$$
\mathcal{L}\left(Z, \partial_{t}\right)=\partial_{t}+\sum_{s=1}^{\infty} w_{s}(Z) \partial_{t}^{-s}
$$


such that for $Z+V t \notin \Sigma$

$$
\mathcal{L}\left(U x+V t+Z, \partial_{t}\right) \psi=k \psi
$$

where $\psi=e^{\left(k t+b x k^{-1}\right)} \phi(U x+Z, t, k)$ is a $\lambda$-periodic solution of 1.7). The coefficients $w_{s}(Z)$ of $\mathcal{L}$ are meromorphic functions on the abelian variety $X$ with poles along the divisor $\Theta$.

Proof. The construction of $\mathcal{L}$ is standard for the KP theory. First, we define $\mathcal{L}$ as a pseudodifferential operator with coefficients $w_{s}(Z, t)$, which are functions of $Z$ and $t$.

Let $\psi$ be a $\lambda$-periodic wave solution. The substitution of (3.18) into (4.2) gives a system of equations that recursively define $w_{s}(Z, t)$ as differential polynomials in $\tilde{\xi}_{s}(Z, t)$. The coefficients of $\psi$ are local meromorphic functions of $Z$, but the coefficients of $\mathcal{L}$ are welldefined global meromorphic functions of on $\mathbb{C}^{g} \backslash \Sigma$, because different $\lambda$-periodic wave solutions are related to each other by $t$-independent factor, which does not affect $\mathcal{L}$. The singular locus is of codimension $\geq 2$. Then Hartogs' holomorphic extension theorem implies that $w_{s}(Z, t)$ can be extended to a global meromorpic function on $\mathbb{C}^{g}$.

The translational invariance of $u$ implies the translational invariance of the $\lambda$-periodic wave solutions. Indeed, for any constant $s$, the series $\phi(V s+Z, t-s, k)$ and $\phi(Z, t, k)$ correspond to $\lambda$-periodic solutions of the same equation. Therefore, they coincide up to a $t$-independent, $\partial_{U}$-invariant factor. This factor does not affect $\mathcal{L}$. Hence, $w_{s}(Z, t)=$ $w_{s}(V t+Z)$.

The $\lambda$-periodic wave functions corresponding to $Z$ and $Z+\lambda^{\prime}$ for any $\lambda^{\prime} \in \Lambda$ are also related to each other by a $t$-independent, $\partial_{U}$-invariant factor. Hence, $w_{s}$ are periodic with respect to $\Lambda$ and therefore are meromorphic functions on the abelian variety $X$. The lemma is proved.

Lemma 4.2 Let $\mathcal{L}$ be a pseudo-differential operator corresponding to $\lambda$-periodic solution and $\mathcal{L}^{*}$ be its formal adjoint operator. Then the following equation

$$
\mathcal{L}^{*}=-\partial_{t} \mathcal{L} \partial_{t}^{-1}
$$

holds.

Recall, that the operator which is formally adjoint to $\left(w \partial^{i}\right)$ is the operator $(-\partial)^{i} \cdot w$, where $w$ stands for the operator of multiplication by the function $w$. Below we will use the notion of the left action of an operator which is identical to the formal adjoint action, i.e. by definition we assume that for a function $f$ the identity

$$
(f \mathcal{D})=\mathcal{D}^{*} f
$$

holds.

Proof. If $\psi$ is as in Lemma 3.3, then there exists a unique pseudo-differential operator $\Phi$ such that

$$
\psi=\Phi e^{k t}, \quad \Phi=1+\sum_{s=1}^{\infty} \varphi_{s}(U x+Z, t) \partial_{t}^{-s} .
$$


The coefficients of $\Phi$ are universal differential polynomials on $\tilde{\xi}_{s}$. Therefore, $\varphi_{s}(z+Z, t)$ is a global meromorphic function of $z \in C^{d}$ and a local meromorphic function of $Z \notin \Sigma$. Note that $\mathcal{L}=\Phi\left(\partial_{t}\right) \Phi^{-1}$, and the equation $H \psi=0$ is equivalent to the operator equation

$$
\partial_{t} \cdot \Phi_{x}+u \Phi=0
$$

where $\Phi_{x}$ is the pseudo-differential operator with the coefficients $\partial_{x} \varphi$. Note that (4.6) implies

$$
\partial_{x} \mathcal{L}=\left[\partial_{t}^{-1} \cdot u, \mathcal{L}\right]
$$

Let us define the dual wave function $\psi^{*}$ by the formula

$$
\psi^{*}=\left(e^{-k t} \partial_{t} \cdot \Phi^{-1} \cdot \partial_{t}^{-1}\right)=\left(\partial_{t}^{-1}\left(\Phi^{-1}\right)^{*} \partial_{t}\right) e^{-k t}
$$

Equation (4.6) implies $H \psi^{*}=0$. The dual wave function $\psi^{*}$ is $\lambda$-periodic. Therefore, the same arguments as used above show that if equation (1.11) is satisfied, then the dual wave function is of the form $\psi^{*}=e^{-\left(k t+b x k^{-1}\right)} \phi^{*}(U x+Z, t, k)$, where the coefficients $\tilde{\xi}_{s}^{*}(z+Z, t)$ of the formal series

$$
\phi^{*}(z+Z, t, k)=1+\sum_{s=1}^{\infty} \tilde{\xi}_{s}^{*}(z+Z, t) k^{-s}
$$

have simple poles at the divisor $\Theta^{U}(t)$. They are $\lambda$-periodic. Therefore,

$$
\phi^{*}(z+Z, t, k)=\phi(z+Z, t,-k) \rho(z+Z, k),
$$

where $\rho$ is a $t$-independent, $\partial_{U}$-invariant factor. Equation (4.10) implies (4.3) and the lemma is proved.

Commuting differential operators. Let as denote strictly positive differential part of the pseudo-differential operator $\mathcal{L}^{m}$ by $\mathcal{L}_{+}^{m}$, i.e. if $\mathcal{L}^{m}=\sum_{i=-m}^{\infty} F_{m}^{(i)} \partial_{t}^{-i}$, then ${ }^{2}$

$$
\mathcal{L}_{+}^{m}=\sum_{i=1}^{m} F_{m}^{(-i)} \partial_{t}^{i}, \quad \mathcal{L}_{-}^{m}=\mathcal{L}^{m}-\mathcal{L}_{+}^{m}=F_{m}^{(0)}+F_{m}^{(1)} \partial_{t}^{-1}+O\left(\partial^{-2}\right)
$$

By definition of the residue of a pseudo-differential operator, the first leading coefficients of $\mathcal{L}_{-}^{m}$ are

$$
F_{m}^{(0)}=\operatorname{res}_{\partial}\left(\mathcal{L}^{m} \partial_{t}^{-1}\right), \quad F_{m}^{(1)}=\operatorname{res}_{\partial} \mathcal{L}^{m}
$$

Lemma 4.3 The operators $\mathcal{L}_{+}^{m}$ satisfy the equations

$$
\begin{aligned}
H \mathcal{L}_{+}^{2 m} & =-F_{2 m, x}^{(0)} \partial_{t}-\frac{1}{2} F_{2 m, x t}^{(0)}+B_{2 m} H, \\
H \mathcal{L}_{+}^{2 m+1} & =-F_{2 m+1, x}^{(1)}+B_{2 m+1} H,
\end{aligned}
$$

where $B_{m}$ is a pseudo-differential operator in the variable $t$.

\footnotetext{
${ }^{2}$ Note that this definition differs from the one used in the KP theory, where plus subscript denotes nonnegative part of a pseudo-differential operator
} 
Proof. First, we prove the equation

$$
H \mathcal{L}_{+}^{m}=-F_{m, x}^{(0)} \partial_{t}-\left(F_{m, x t}^{(0)}+F_{m, x}^{(1)}\right)+B_{m} H
$$

Each operator $\mathcal{D}$ of the form $\mathcal{D}=\sum_{i=N}^{\infty}\left(a+b \partial_{x}\right) \partial_{t}^{-i}$ can be uniquely represented in the form $\mathcal{D}=D_{1}+D_{2} H$, where $D_{1,2}$ are pseudo-differential operators in the variable $t$. Consider such a representation for the operator $H \mathcal{L}^{m}=D_{1}+D_{2} H$. From the definition of $\mathcal{L}$ it follows that $H \mathcal{L}^{m} \psi=0$. That implies $D_{1}=0$ or the equation

$$
H \mathcal{L}^{m}=D_{2} H
$$

We have the identity

$$
\left[\partial_{x} \partial_{t}+u, \mathcal{L}_{+}^{m}\right]=\mathcal{L}_{+, x t}^{m}+\mathcal{L}_{+, x}^{m} \partial_{t}+\left[u, \mathcal{L}_{+}^{m}\right]-\mathcal{L}_{+, t}^{m} \partial_{t}^{-1} \cdot u+\mathcal{L}_{+, t}^{m} \cdot \partial_{t}^{-1} \cdot H
$$

The first three terms are differential operators in the $t$ variable. By definition of $\mathcal{L}_{+}^{m}$ the fourth term is also a differential operator. Therefore, the pseudo-differential operator $D_{1}$ in the decomposition $H \mathcal{L}_{+}^{m}=D_{m, 1}+B_{m} H$ is a differential operator.

In the same way we get the equation

$$
H \mathcal{L}_{-}^{m}=\tilde{D}_{m, 1}+\tilde{B}_{2 m} H
$$

where

$$
\tilde{D}_{m, 1}=\mathcal{L}_{-, x t}^{m}+\mathcal{L}_{-, x}^{m} \partial_{t}+\left[u, \mathcal{L}_{-}^{m}\right]-\mathcal{L}_{-, t}^{m} \partial_{t}^{-1} \cdot u
$$

By definition of $\mathcal{L}_{-}^{m}$ the operator $\tilde{D}_{m, 1}$ is a pseudo-differential operator of order not greater than 1. Equation (4.16) implies $H \mathcal{L}_{+}^{m}=-H \mathcal{L}_{-}^{m}+D_{2} H$. Hence, $D_{m, 1}=-\tilde{D}_{m, 1}$ is a differential operator of the order 1, i.e. has the form $a \partial_{t}+b$. The coefficients of this operator can be easily found from the leading coefficients of the right hand side of (4.19). Direct computations give equation (4.15).

Now in order to complete the proof of (4.13) and (4.14) it is enough to use (4.3). From equation (4.3) and the relation $\operatorname{res}_{\partial} D=-\operatorname{res}_{\partial} D^{*}$ it follows that

$$
F_{2 m}^{(1)}=-\operatorname{res}_{\partial}\left(\mathcal{L}^{*}\right)^{2 m}=-\operatorname{res}_{\partial}\left(\partial_{t} \mathcal{L}^{m} \partial_{t}^{-1}\right)=-F_{2 m, t}^{(0)}-F_{2 m}^{(1)}
$$

In the same way we get

$$
F_{2 m+1}^{(0)}=\operatorname{res}_{\partial}\left(\mathcal{L}^{2 m+1} \partial_{t}^{-1}\right)=\operatorname{res}_{\partial}\left(\mathcal{L}^{2 m+1} \partial_{t}^{-1}\right)^{*}=-F_{2 m+1}^{(0)}=0 .
$$

Equations (4.15, 4.20, 4.21) imply (4.13) and (4.14). The lemma is proved.

The following statement is a direct corollary of equations (4.20, 4.21).

Corollary 4.1 The operators $\mathcal{L}_{+}^{m}$ satisfy the relation

$$
\left(\mathcal{L}_{+}^{m}\right)^{*}=(-1)^{m} \partial_{t} \cdot \mathcal{L}_{+}^{m} \cdot \partial_{t}^{-1}
$$


The next step is crucial for the construction of commuting operators.

Lemma 4.4 The functions $F_{2 m}^{(0)}, F_{2 m+1}^{(1)}$ have at most second order pole on the divisor $\Theta$.

Proof. The ambiguity in the definition of $\psi$ does not affect the product

$$
\psi^{*} \psi=\left(e^{-k t} \partial_{t} \Phi^{-1} \partial_{t}^{-1}\right)\left(\Phi e^{k t}\right)
$$

Therefore, although each factor is only a local meromorphic function on $\mathbb{C}^{g} \backslash \Sigma$, the coefficients $J_{s}^{(0)}$ of the product

$$
\psi^{*} \psi=\phi^{*}(Z, t, k) \phi(Z, t, k)=1+\sum_{s=2}^{\infty} J_{s}^{(0)}(Z, t) k^{-s}
$$

are global meromorphic functions of $Z$. Moreover, the translational invariance of $u$ implies that they have the form $J_{s}(Z, t)=J_{s}(Z+V t)$. Each of the factors in the left hand side of (4.24) has a simple pole on $\Theta-V t$. Hence, $J_{s}(Z)$ is a meromorphic function on $X$ with a second order pole at $\Theta$.

From the definition of $\mathcal{L}$ it follows that

$$
\operatorname{res}_{k}\left(\psi^{*}\left(\mathcal{L}^{n} \psi\right)\right) k^{-1} d k=\operatorname{res}_{k}\left(\psi^{*} k^{n} \psi\right) k^{-1} d k=J_{n}^{(0)} .
$$

On the other hand, using the identity

$$
\operatorname{res}_{k}\left(e^{-k x} \mathcal{D}_{1}\right)\left(\mathcal{D}_{2} e^{k x}\right) d k=\operatorname{res}_{\partial}\left(\mathcal{D}_{2} \mathcal{D}_{1}\right)
$$

we get

$$
\operatorname{res}_{k}\left(\psi^{*}\left(\mathcal{L}^{n} \psi\right)\right) k^{-1} d k=\operatorname{res}_{k}\left(e^{-k t} \partial_{t} \Phi^{-1} \partial_{t}^{-1}\right)\left(\mathcal{L}^{n} \Phi \partial_{t}^{-1} e^{k t}\right) d k=\operatorname{res}_{\partial}\left(\mathcal{L}^{n} \partial_{t}^{-1}\right)=F_{n}^{(0)}
$$

Therefore, $F_{n}^{(0)}=J_{n}^{(0)}$ has the second order pole at $\Theta$.

Consider now the coefficients $J_{s}^{(1)}$ of the series

$$
\psi^{*} \psi_{t}-\psi_{t}^{*} \psi=2 k+\sum_{s=1}^{\infty} 2 J_{s}^{(1)}(Z, t) k^{-s} .
$$

They are meromorphic functions on $X$ with the second order pole at $\Theta$. We have

$$
2 J_{n}^{(1)}=\operatorname{res}_{k}\left(\left(\psi^{*} \mathcal{L}^{n}\right) \psi_{t}-\psi_{t}^{*}\left(\mathcal{L}^{n} \psi\right)\right) k^{-1} d k=\operatorname{res}_{\partial}\left(\mathcal{L}^{n}+\partial_{t} \mathcal{L}^{n} \partial_{t}^{-1}\right)=2 F_{n}^{(1)}+F_{n, t}^{(0)}
$$

Then from equation (4.21) it follows that $F_{2 m+1}^{(1)}=J_{2 m+1}^{(1)}$ and the lemma is proved.

Let $\mathbf{F}$ be a direct sum of the linear spaces $\hat{\mathbf{F}}^{\alpha}, \alpha=0,1$, spanned by $\left\{F_{2 m+\alpha}^{(\alpha)}, m=0,1, \ldots\right\}$. They are subspaces of the $2^{g}$-dimensional space of the abelian functions with at most second order pole at $\Theta$. Therefore, for all but $\hat{g}^{\alpha}=\operatorname{dim} \hat{\mathbf{F}}^{\alpha}$ positive integers $2 n+\alpha$, there exist constants $c_{i, n}^{(\alpha)}$ such that

$$
F_{2 n+\alpha}^{(\alpha)}(Z)+\sum_{i=1}^{n} c_{i, n}^{(\alpha)} F_{2 n-2 i+\alpha}^{(\alpha)}(Z)=0 .
$$

Let $I^{(\alpha)}$ denote the subset of integers $2 n+\alpha$ for which none of such constants exist. We call the union $I=I^{(0)} \cup I^{(1)}$ the gap sequence. 
Lemma 4.5 Let $\mathcal{L}$ be the pseudo-differential operator corresponding to a $\lambda$-periodic wave function $\psi$ constructed above. Then, for the differential operators

$$
L_{2 n+\alpha}=\mathcal{L}_{+}^{2 n+\alpha}+\sum_{i=1}^{n} c_{i, n}^{(\alpha)} \mathcal{L}_{+}^{2 n+\alpha-2 i}, \quad 2 n+\alpha \notin I^{\alpha},
$$

the equations

$$
L_{2 n+\alpha} \psi=a_{2 n+\alpha}(k) \psi, \quad a_{2 n+\alpha}(k)=k^{2 n+\alpha}+\sum_{s=1}^{\infty} a_{s, n} k^{2 n+\alpha-s},
$$

where $a_{s, n}$ are constants, hold.

Proof. First note, that from (4.13, 4.14) it follows that $H L_{2 n+\alpha} \psi=0$. Hence, if $\psi$ is a $\lambda$-periodic wave solution of (1.7) corresponding to $Z \notin \Sigma$, then $L_{2 n+\alpha} \psi$ is also a formal solution of the same equation. That implies the equation $L_{2 n+\alpha} \psi=a_{2 n+\alpha}(Z, k) \psi$, where $a_{2 n+\alpha}(Z, k)$ is $t$-independent and $\partial_{U}$-invariant function of the variable $Z$. The ambiguity in the definition of $\psi$ does not affect $a_{2 n+\alpha}$. Therefore, the coefficients of $a_{2 n+\alpha}$ are well-defined global meromorphic functions on $\mathbb{C}^{g} \backslash \Sigma$. The $\partial_{U^{-}}$invariance of $a_{2 n+\alpha}$ implies that $a_{2 n+\alpha}$, as a function of $Z$, is holomorphic outside the locus. Hence it has an extension to a holomorphic function on $\mathbb{C}^{g}$. The $\lambda$-periodic wave functions corresponding to $Z$ and $Z+\lambda^{\prime}$ for any $\lambda^{\prime} \in \Lambda$ are related to each other by a $t$-independent, $\partial_{U}$-invariant factor. Hence, $a_{2 n+\alpha}$ is periodic with respect to $\Lambda$ and therefore is $Z$-independent. Note that $a_{2 s+1, n}=0$ and $a_{2 s, n}=c_{s, n}$ if $s \leq n$. The lemma is proved.

The operator $L_{m}$ can be regarded as a $(Z, x)$-parametric family of ordinary differential operators $L_{m}^{Z}$ whose coefficients have the form

$$
L_{m}^{Z, x}=\partial_{t}^{m}+\sum_{i=1}^{m} u_{i, m}(U x+V t+Z) \partial_{t}^{m-i}, \quad m \notin I .
$$

where $u_{i, m}(Z)$ are abelian function regular outside of $\Theta$. For $Z+U x \notin \Sigma_{-}$the coefficients of $L_{m}^{Z, x}$ are meromorphic functions of the variable $t$, which are not identically equal infinity. Recall, that $\Sigma_{-}$is a $\partial_{V}$-invariant set of $\Theta$.

Corollary 4.2 The operators $L_{m}^{Z, x}$ commute with each other,

$$
\left[L_{n}^{Z, x}, L_{m}^{Z, x}\right]=0
$$

From (4.32) it follows that $\left[L_{n}^{Z, x}, L_{m}^{Z, x}\right] \psi=0$. The commutator is an ordinary differential operator. Hence, the last equation implies (4.34).

Lemma 4.6 Let $\mathcal{A}^{Z, x}, Z+U x \notin \Sigma_{-}$, be a commutative ring of ordinary differential operators spanned by the operators $L_{n}^{Z, x}$. Then there is an irreducible algebraic curve $\Gamma$ such that $\mathcal{A}^{Z, x}$ is isomorphic to the ring $A_{-}\left(\Gamma, P_{+}, P_{-}\right)$of the meromorphic functions on $\Gamma$ with the only pole at a smooth point $P_{-}$vanishing at another smooth point $P_{+}$. The correspondence 
$Z \rightarrow \mathcal{A}^{Z, 0}$ defines a holomorphic map of $X \backslash \Sigma_{-}$into the space of torsion-free rank 1 sheaves $\mathcal{F}$ on $\Gamma$

$$
j: X \backslash \Sigma_{-} \longmapsto \overline{\operatorname{Pic}}(\Gamma) .
$$

On an open set the map $j$ is an imbedding.

The proof of the lemma is almost identical to the proof of lemma 3.4 in [7]. It is the fundamental fact of the theory of commuting linear ordinary differential operators ([12, 13, 14, 15, 21] ) that there is a natural correspondence

$$
\mathcal{A} \longleftrightarrow\left\{\Gamma, P_{-},\left[k^{-1}\right]_{1}, \mathcal{F}\right\}
$$

between regular at $t=0$ commutative $\operatorname{rings} \mathcal{A}$ of ordinary linear differential operators in the variable $t$, containing a pair of monic operators of co-prime orders, and sets of algebraicgeometrical data $\left\{\Gamma, P_{-},\left[k^{-1}\right]_{1}, \mathcal{F}\right\}$, where $\Gamma$ is an algebraic curve with a fixed first jet $\left[k^{-1}\right]_{1}$ of a local coordinate $k^{-1}$ in the neighborhood of a smooth point $P_{-} \in \Gamma$ and $\mathcal{F}$ is a torsionfree rank 1 sheaf on $\Gamma$ such that

$$
H^{0}(\Gamma, \mathcal{F})=H^{1}(\Gamma, \mathcal{F})=0 .
$$

The correspondence becomes one-to-one if the rings $\mathcal{A}$ are considered modulo conjugation $\mathcal{A}^{\prime}=g(t) \mathcal{A} g^{-1}(t)$.

Note, that in [12, 13, 14, 15, the main attention was paid to the generic case of the commutative rings corresponding to smooth algebraic curves. The invariant formulation of the correspondence given above is due to Mumford [21].

The algebraic curve $\Gamma$ is called the spectral curve of $\mathcal{A}$. The $\operatorname{ring} \mathcal{A}$ is isomorphic to the ring $A\left(\Gamma, P_{-}\right)$of meromorphic functions on $\Gamma$ with the only pole at the puncture $P_{-}$. The isomorphism is defined by the equation

$$
L_{a} \psi_{0}=a \psi_{0}, \quad L_{a} \in \mathcal{A}, a \in A\left(\Gamma, P_{-}\right) \text {. }
$$

Here $\psi_{0}$ is a common eigenfunction of the commuting operators. At $t=0$ it is a section of the sheaf $\mathcal{F} \otimes \mathcal{O}\left(-P_{-}\right)$.

Important remark. The construction of the correspondence (4.36) depends on a choice of the initial point $t_{0}=0$. The spectral curve and the sheaf $\mathcal{F}$ are defined by the evaluations of the coefficients of generators of $\mathcal{A}$ and a finite number of their derivatives at the initial point. In fact, the spectral curve is independent on the choice of $t_{0}$, but the sheaf does depend on it, i.e. $\mathcal{F}=\mathcal{F}_{t_{0}}$.

Using the shift of the initial point it is easy to show that the correspondence (4.36) extends to the commutative rings of operators whose coefficients are meromorphic functions of $t$ at $t=0$. The rings of operators having poles at $t=0$ correspond to sheaves for which the condition (4.37) is violated.

As it was mentioned above, the operators $L_{n}, L_{m}$ can be seen as a $(Z, x)$-parametric family of commuting ordinary differential operators in the variable $t$. Let $\Gamma^{Z, x}$ be the corresponding spectral curve. The eigenvalues $a_{n}(k)$ of the operators $L_{n}^{Z, x}$ defined in (4.32) coincide with 
the Laurent expansions at $P_{-}$of the meromorphic functions $a_{n} \in A\left(\Gamma^{Z, x}, P_{-}\right)$. They are $(Z, x)$-independent. Hence, the spectral curve is $(Z, x)$-independent, as well, $\Gamma=\Gamma^{Z, x}$.

Equations (1.10), which are equivalent to (1.11) and are sufficient for the construction of the $\lambda$-periodic wave solutions, are symmetric with respect to $x$ and $t$. Therefore, the simple interchange of the variables $x$ and $t$ shows that if equations (1.10) hold then there exist commuting ordinary differential operators $L_{m}^{+}$of the form

$$
L_{m}^{+}=\partial_{x}^{m}+\sum_{i=1}^{m} u_{i, m}^{+}(U x+V t+Z) \partial_{x}^{m-i}, \quad m \notin I^{+},
$$

where $I^{+}$is the gap sequence associated with the variable $x$. These operators satisfy the equations

$$
L_{m}^{+} H=B_{m}^{+} H
$$

where $B_{m}^{+}$are differential operators in the variable $x$.

Let $\psi$ be a $\lambda$-periodic solution of (1.7). Then the same arguments as in the proof of Lemma 4.5 show that equations (4.40) imply

$$
L_{n}^{+} \psi=a_{n}^{+}(k) \psi, \quad a_{n}^{+}=\sum_{s=1}^{\infty} a_{s, n}^{+} k^{-s}
$$

where $a_{s, n}^{+}$are constants. From (4.41) it follows that the operators $L_{n}, L_{m}^{+}$satisfy the equation

$$
\left[L_{n}, L_{m}^{+}\right]=B H
$$

where $B$ is a differential operator in the variables $x, t$. Equation (4.41) also implies that there exists a polynomial $\tilde{R}$ such that $\tilde{R}\left(L_{n}, L_{m}^{+}\right) \psi=0$, i.e. eigenvalues $a_{n}, a_{m}^{+}$of $L_{n}$ and $L_{m}^{+}$satisfy the equation $\tilde{R}\left(a_{n}, a_{m}^{+}\right)=0$. Therefore, the spectral curves of commutative rings $\mathcal{A}^{Z}$ and $\mathcal{A}_{+}^{Z}$ coincide. Note, that (4.41) implies that $a_{m}^{+}$vanishes at $P_{-}$. The symmetry between $x$ and $t$ variables implies that the $\operatorname{ring} \mathcal{A}_{+}^{Z}$ is isomorphic to the $\operatorname{ring} A_{+}\left(\Gamma, P_{+}, P_{-}\right)$ of meromorphic functions on $\Gamma$ with the only pole at $P_{+}$that vanish at $P_{-}$.

Let us fix $x=0$ and consider the commuting operators $L_{n}^{Z}=L_{n}^{Z, 0}$. The construction of the correspondence (4.36) implies that if the coefficients of the operators in $\mathcal{A}$ holomorphically depend on parameters, then the algebraic-geometrical spectral data are also holomorphic functions of the parameters.

Therefore, $j$ is holomorphic out of $\Theta$. Then, using the shift of the initial point and the fact, that $\mathcal{F}_{t_{0}}$ holomorphically depends on $t_{0}$, we get that $j$ holomorphically extends on $\Theta \backslash \Sigma_{-}$, as well.

The theta-divisor is not invariant under the shifts by constant vectors. Hence, for the generic $Z$ and $Z^{\prime}$ the operators in $\mathcal{A}^{Z}$ and $\mathcal{A}^{Z^{\prime}}$ have different poles. Hence, those rings do not coincide. Thus, the map $j$ is an imbedding on an open set. The lemma is proved.

It implies the global existence of the wave function. 
Lemma 4.7 Let equations (1.10) hold. Then there exists a common eigenfunction of the operators $L_{n}^{Z}$ of the form $\psi=e^{k t} \phi(V t+Z, k)$ such that the coefficients of the formal series

$$
\phi(Z, k)=1+\sum_{s=1}^{\infty} \xi_{s}(Z) k^{-s}
$$

are global meromorphic functions with a simple pole at $\Theta$.

The proof of the lemma is identical to the proof of lemma $3.5 \mathrm{in}[7$. The function $\psi$ is first defined for $Z \notin \Sigma_{-}$as the inverse image $\psi=j^{*} \hat{\psi}_{B A}$ of the Baker-Akhiezer function, which is known to be globally defined on $\overline{\operatorname{Pic}}(\Gamma)$. Then, Hartogs' extension theorem implies that $\psi$ has a meromorphic extension on $C^{g}$. The Baker-Akhiezer function is regular out of divisor corresponding to the commutative rings of operators whose coefficients have poles at $t=0$. Hence, $\psi$ is holomorphic out of $\Theta$.

Let us show now that the correspondence $\psi \rightarrow \psi^{*}$ defines an involution of $\Gamma$ under which $P_{ \pm}$are fixed.

Lemma 4.8 The eigenvalues $a_{2 n+\alpha}$ of the commuting operators $L_{2 n+\alpha}$ satisfy the relation

$$
a_{2 n+\alpha}(k)=(-1)^{\alpha} a_{2 n+\alpha}(-k) .
$$

Proof. From equations (4.104.224.31) it follows that

$$
\begin{gathered}
\psi_{t}^{*}\left(L_{2 n+\alpha} \psi\right)=a_{2 n+\alpha}(k)\left(\psi_{t}^{*} \psi\right), \\
\left(\psi_{t}^{*} L_{2 n+\alpha}\right) \psi=\left(\left(L_{2 n+\alpha}^{*} \psi_{t}^{*}\right) \psi\right)=(-1)^{\alpha} a_{2 n+\alpha}(-k)\left(\psi_{t}^{*} \psi\right) .
\end{gathered}
$$

The left and right action of pseudo-differential operators are formally adjoint, i.e., for any two operators the equality $\left(e^{-k t} \mathcal{D}_{1}\right)\left(\mathcal{D}_{2} e^{k t}\right)=e^{-k t}\left(\mathcal{D}_{1} \mathcal{D}_{2} e^{k t}\right)+\partial_{t}\left(e^{-k t}\left(\mathcal{D}_{3} e^{k t}\right)\right)$ holds. Here $\mathcal{D}_{3}$ is a pseudo-differential operator whose coefficients are differential polynomials in the coefficients of $\mathcal{D}_{1}$ and $\mathcal{D}_{2}$. Therefore, equations (4.454.46) imply

$$
\left(a_{2 n+\alpha}(k)-(-1)^{\alpha} a_{2 n+\alpha}(-k)\right)\left(\psi_{t}^{*} \psi\right)=\partial_{t} Q_{2 n+\alpha} .
$$

The coefficients of the series $Q_{2 n+\alpha}$ are differential polynomials on the coefficients of the wave operator $\Phi$ defined by equation (4.5). For the globally defined wave function $\psi$, which exists according to the previous lemma, the coefficients of the wave operator are global meromorphic functions. Hence,

$$
Q_{2 n+\alpha}=\sum_{s=1}^{\infty} Q_{2 n+\alpha, s}(V t+Z),
$$

where $Q_{2 n+\alpha, s}(Z)$ are meromorphic functions regular out of $\Theta$.

In a similar way we have

$$
\psi_{t}^{*} \psi=\left(e^{-k t} \partial_{t} \Phi^{-1}\right)\left(\Phi e^{k t}\right)=k+\partial_{t} Q^{(1)}
$$


The series $Q^{(1)}$ has the form

$$
Q^{(1)}=\sum_{s=1}^{\infty} Q_{s}^{(1)}(V t+Z)
$$

where $Q_{s}^{(1)}(Z)$ are meromorphic functions regular out of $\Theta$.

Let us fix a neighborhood of the theta-divisor in $X$. It defines the neighborhood $S$ of $\Theta$ in $\mathbb{C}^{\widehat{g}}$. Outside of $S$ the functions $Q_{s}^{(1)}$ are bounded. Consider a sequence of real numbers $l_{i} \rightarrow \infty$ such that $Z \pm V l_{i}$ is not in $S$. Then, from (4.49) it follows that

$$
\left\langle\psi_{t}^{*} \psi\right\rangle=\lim _{l_{i} \rightarrow \infty} \frac{1}{2 l_{i}} \int_{-l_{i}}^{l_{i}}\left(\psi_{t}^{*} \psi\right) d t=k .
$$

The integration (4.51) is taken along a curve connecting points $Z+ \pm V l_{i}$ and which does not intersect $\Theta$.

The same arguments imply that under "averaging" in $t$ the right hand side of (4.47) vanishes. Hence, (4.47) implies (4.44). The lemma is proved.

The series $a_{n}(k)$ are the expansions at $P_{-}$of meromorphic functions on $\Gamma$. Therefore, from (4.44) it follows there exists a holomorphic involution $\sigma: \Gamma \rightarrow \Gamma$ of the spectral curve such that

$$
a_{n}^{\sigma}=a_{n}(\sigma(P))=(-1)^{n} a_{n}(P)
$$

The point $P_{-}$is fixed under $\sigma$ and the local parameter is odd with respect to $\sigma$, i.e. $\sigma^{*} k=-k$. In the same way using $x$ variable instead of $t$ we get that the second puncture $P_{+} \in \Gamma$ is also fixed under $\sigma$.

The involution $\sigma$ induces an involution on the generalized Jacobian $J(\Gamma)$ which is by definition is the group of the equivalence classes of zero-degree divisors on $\Gamma$, i.e. $J(\Gamma)=$ $\operatorname{Pic}^{0}(\Gamma)$. The odd subgroup of $J(\Gamma)$ with respect to the induced involution $\sigma^{*}$ is the Prym variety of the spectral curve, $\mathcal{P}(\Gamma)=\operatorname{ker}\left(1+\sigma^{*}\right)$. Our next goal is to show that $\mathcal{P}(\Gamma)$ of the spectral curve is compact.

Lemma 4.9 There exist $g$-dimensional vectors $V_{2 m+1}=\left\{V_{2 m+1, k}\right\}$ and constants $v_{2 m+1}$ such that

$$
F_{2 m+1}^{(1)}(Z)=\sum_{k=1}^{g} V_{2 m+1, k} \partial_{V} h_{k}(Z)+v_{2 m+1},
$$

where $F_{2 m+1}^{(1)}=\operatorname{res}_{\partial} \mathcal{L}^{2 m+1}$ and $h_{k}=\partial_{z_{k}} \ln \theta(Z)$.

Proof. From equations (4.28, 4.29) and (4.49) it follows that $F_{2 m+1}^{(1)}=-2 \partial_{V} Q_{2 m+1}^{(1)}$, where $Q_{2 m+1}^{(1)}$ is a meromorphic function with a pole along $\Theta$. The function $F_{2 m+1}^{(1)}$ is an abelian function. Hence, for any vector $\lambda$ in the period lattice $Q_{2 m+1}^{(1)}(Z+\lambda)=Q_{2 m+1}^{(1)}(Z)+c_{m, \lambda}$. There is no abelian function with a simple pole on $\Theta$. Hence, there exists a constant $q_{n}$ and $g$-dimensional vectors $l_{m}$ and $V_{2 m+1}$, such that $Q_{2 m+1}^{(1)}=q_{m}+\left(l_{m}, Z\right)+\left(V_{2 m+1}, h(Z)\right)$, where $h(Z)$ is a vector with the coordinates $h_{k}(Z)$. Therefore, $F_{2 m+1}^{(1)}=\left(l_{m}, V\right)+\left(V_{2 m+1}, \partial_{V} h(Z)\right)$. The lemma is proved. 
In order to complete the proof of our main result we need few more facts of the KP theory: flows of the KP hierarchy define deformations of the commutative rings $\mathcal{A}$ of ordinary linear differential operators. The spectral curve is invariant under these flows. If a commutative ring $\mathcal{A}$ of linear ordinary differential operators is maximal, i.e., it is not contained in any bigger commutative ring, then the KP orbit of $\mathcal{A}$ is isomorphic to the generalized Jacobian $J(\Gamma)=\operatorname{Pic}^{0}(\Gamma)$ of the spectral curve of $\mathcal{A}$ (see details in [3, 12, 13, 22]).

The KP hierarchy in the Sato form is a system of commuting differential equation for a pseudo-differential operator $\mathcal{L}$

$$
\partial_{t_{n}} \mathcal{L}=\left[\mathcal{L}_{+}^{n}, \mathcal{L}\right]
$$

If the operator $\mathcal{L}$ is as above. i.e., if it is defined by $\lambda$-periodic wave solutions of equation (1.7), then equation (4.14) implies that for odd $n$ equations (4.54) are equivalent to the equations

$$
\partial_{t_{2 n+1}} u=\partial_{x} F_{2 m+1}^{(1)}(U x+V t+Z) .
$$

The first time of the hierarchy is identified with the variable $t_{1}=t$.

Equations (4.55) identify the space generated by the functions $\partial_{U} F_{2 m+1}^{(1)}$ with the tangent space at $\mathcal{A}^{Z}$ of the orbit of the part of the NV hierarchy associated with the puncture $P_{-}$. In terms of $u$ the deformation with respect to $z_{i}$ is given by the equation

$$
\partial_{z_{i}} u=\partial_{x} \partial_{V} h_{i}, \quad h_{i}=\partial_{z_{i}} \ln \theta(Z) .
$$

Equations (4.55, 4.56) and (4.53) imply

$$
\partial_{t_{2 n+1}}=\partial_{V_{2 n+1}}=\sum_{k=1}^{g} V_{2 n+1, k} \partial_{z_{k}} .
$$

Hence, the orbit of $\mathcal{A}^{Z}$ is isomorphic to the factor of $Z+Y / T(Z)$ of the affine subvariety $Z+Y \subset X$, where $Y$ is the closure in $X$ of the subgroup $\left\langle\sum_{n} V_{2 n+1} t_{2 n+1}\right\rangle$, and $T(Z)$ is a lattice in the universal cover of $Y$.

Lemma 4.10 For the generic $Z \notin \Sigma_{-}$, the orbit of $\mathcal{A}^{Z}$ under the $N V$ flows defines an isomorphism:

$$
i_{Z}: \mathcal{P}(\Gamma) \longmapsto Z+Y \subset X
$$

Proof. Recall, that according to [5] the NV orbit of a maximal commutative ring is isomorphic to the Prym variety of the corresponding spectral curve. The arguments showing that $\mathcal{A}^{Z}$ is maximal for the generic $Z$ are identical to those used in [7]. Indeed, suppose that $\mathcal{A}^{Z}$ is not maximal for all $Z$. Then there exits $2 n+\alpha \in I$, where $I$ is the gap sequence defined above, such that for each $Z \notin \Sigma_{-}$there exists an operator $L_{2 n+\alpha}^{Z}$ of order $2 n+\alpha$ which commutes with all the operators $L_{m}^{Z} \in \mathcal{A}^{Z}$. Therefore, it commutes with $\mathcal{L}$. That implies the equality

$$
F_{2 n+\alpha}^{(\alpha)}(Z)+\sum_{i=1}^{n} c_{i, n}^{(\alpha)}(Z) F_{2 n-2 i+\alpha}^{(\alpha)}(Z)=0 .
$$


Note the difference between (4.30) and (4.59). In the first equation the coefficients $c_{i, n}^{(\alpha)}$ are constants.

The $\lambda$-periodic wave solution of equation (1.7) is a common eigenfunction of all commuting operators, i.e. $L_{2 n+\alpha} \psi=a_{2 n+\alpha}(Z, k) \psi$, where is $\partial_{V}$-invariant. The compactness of $X$ implies that $a_{2 n+\alpha}$ is $Z$-independent. The first $n$ coefficients of $a_{2 n+\alpha}$ coincide with the coefficients in (4.59). Hence, these coefficients are $Z$-independent. That contradicts the assumption that $2 n+\alpha \in I$.

The map $j$ defined in Lemma 4.6 restricted to $Z+Y \subset X$ is inverse to $i_{Z}$. For the generic $Z$ it is an imbedding. Hence for the generic $Z$ the lattice $T(Z)$ is trivial. The lemma is thus proven.

Corollary 4.3 The Prym variety $\mathcal{P}(\Gamma)$ of the spectral curve $\Gamma$ is compact.

The compactness of the Prym variety is not as restrictive, as the compactness of the Jacobian (see 24]). Nevertheless, it implies an explicit description of the the singular points of the spectral curve. The proof of the following statement is due to Robert Friedman and is presented in the Appendix.

Corollary 4.4 (R. Friedman) The spectral curve $\Gamma$ is smooth outside of fixed points $P_{ \pm}, Q_{k}$ of the involution $\sigma$. The branches of $\Gamma$ at $Q_{k}$ are linear and are not permuted by $\sigma$.

An equivalent formulation of the corollary is as follows: there is a a smooth algebraic curve $\tilde{\Gamma}$ with involution $\tilde{\sigma}$ and a regular equivariant map $p: \tilde{\Gamma} \rightarrow \Gamma$ which is one-to-one out of preimages $Q_{k}^{i}, i=1 \ldots, \nu_{k}$, on $\tilde{\Gamma}$ of the singular points $Q_{k}$.

The common eigenfunction of commuting differential operators is well-defined up to a constant factor for all smooth points of the spectral curve. It can be analytically extended along the branches of the spectral curve passing through the singular points, i.e. the preimage $\tilde{\psi}$ of the Baker-Akhiezer on $\tilde{\Gamma}$ can be regarded as a section of a line bundle on $\tilde{\Gamma}$. From the construction of the correspondence (4.36) it follows that the evaluations of $\tilde{\psi}$ at the preimages of the singular points $Q_{k}$ satisfy linear relations

$$
\sum_{i=1}^{\nu_{k}} c_{k, j}^{i} \tilde{\psi}\left(t, Q_{k}^{i}\right)=0, \quad j=1, \ldots, n_{k}
$$

The coefficients of these relations and the zero divisor $D$ of $\tilde{\psi}(0, \tilde{P})$ can be regarded the data defining the corresponding sheaf $\mathcal{F}$. The divisor $D$ is the pole divisor of the normalized eigenfunction $\tilde{\psi}_{0}(t, \tilde{P})=\tilde{\psi}(t, \tilde{P}) / \tilde{\psi}(0, \tilde{P})$.

The following theta-functional formula (4.64) for $\tilde{\psi}$ is crucial for the final steps of the proof. First note that using the transformation $\psi \longmapsto e^{(l(k), Z)} \psi$, where $l(k)$ is a series such that $(l(k), V)=0$, we may assume without loss of generality that the series $\phi$ in (4.43) satisfies the following monodromy properties:

$$
\phi\left(Z+e_{j}, k\right)=\phi(Z), \quad \phi\left(Z+B_{j}\right)=\phi(Z) \rho_{j}(k)
$$


where $e_{j}$ are the basis vectors in $\mathbb{C}^{g}$ and $B_{j}$ are vectors defined by the columns of the matrix $B$, corresponding to the principle polarization of $X$.

Equations (4.61) and the fact the the coefficients of $\phi$ are meromorphic functions with simple poles along $\Theta$ imply that there is a series $A(k)$ such that

$$
\phi=\frac{\theta(A(k)+Z)}{\theta(Z)}
$$

The series $A(k)$ defines an imbedding of the neighborhood of $P_{-}$into $X$.

The same arguments show that there is a holomorphic map

$$
\tilde{A}: \tilde{\Gamma} \longmapsto X
$$

such that the function $\tilde{\psi}(t, \tilde{P}), \tilde{P} \in \tilde{\Gamma}$, given by the formula

$$
\tilde{\psi}=\frac{\theta(\tilde{A}(\tilde{P})+V t+Z)}{\theta(V t+Z)} e^{t \Omega(\tilde{P})},
$$

is the common eigenfunction of the operators in $\mathcal{A}^{Z}$. Here $\Omega(\tilde{P})$ is an abelian integral on $\tilde{\Gamma}$ having the form $\Omega=k+O\left(k^{-1}\right)$ at $P_{-}$. Then the normalized eigenfunction of the commuting operators is given by the formula

$$
\tilde{\psi}_{0}=\frac{\theta(\tilde{A}(\tilde{P})+V t+Z) \theta(Z)}{\theta(\tilde{A}(\tilde{P})+Z) \theta(V t+Z)} e^{t \Omega(\tilde{P})} .
$$

Our next goal is to show that the pole divisor $D$ of $\tilde{\psi}_{0}$ satisfies the condition analogous to (2.15) found by Novikov and Veselov in the case of the smooth spectral curves.

Lemma 4.11 The equivalence class of $[D] \in J(\tilde{\Gamma})$ of the divisor $D$ satisfies the equation

$$
[D]+[\tilde{\sigma}(D)]=K+P_{+}+P_{-}+\sum_{k, i} Q_{k}^{i} \in J(\tilde{\Gamma}),
$$

where $K$ is the canonical class, i.e. the equivalence class of the zero-divisor of a holomorphic differential on $\tilde{\Gamma}$.

Proof. Equation (4.66) is equivalent to the condition that the divisor $D+\sigma(D)$ is the zero divisor of a meromorphic differential $d \Omega$ on $\tilde{\Gamma}$ with simple poles at the punctures $P_{ \pm}$and the points $Q_{k}^{i}$. The differential $d \Omega$ is even with respect to the involution and descends to a meromorphic differential on the factor-curve $\Gamma_{0}$.

The existence of such differential can be proved almost identically to the proof of the statement that the conditions (2.15) are necessary conditions for the potential reduction of the $2 D$ Schrödinger operators given in [17] (Theorem 3.1).

Let $\tilde{\psi}_{0}(x, t, P)$ be the normalized solution of the Schrödinger operator. It is obtained by the deformation along the $x$-flow from the normalized eigenfunction of the operators in 
$\mathcal{A}^{Z}$ considered above. Therefore, it has the form (2.16) with $\theta_{p r}$ and $A^{p r}$ replaced by $\theta$ and $\tilde{A}$, respectively. Following [23] we present another real form of $\tilde{\psi}_{0}$. Let us introduce real coordinates of a complex vector $Z \in \mathbb{C}^{g}$ by the formula $Z=\zeta^{\prime}+B \zeta^{\prime \prime}$, where $\zeta^{\prime}, \zeta^{\prime \prime}$ are $g$-dimensional real vectors, and $B$ is the matrix of $b$-periods of the normalized holomorphic differentials on $\Gamma$. Then the absolute value $|\phi|$ of the function $\phi(\zeta, P), \zeta=\left(\zeta^{\prime}, \zeta^{\prime \prime}\right)$ given by the formula

$$
\phi(\zeta, P)=\frac{\theta(\tilde{A}(P)+Z)}{\theta(Z)} e^{2 \pi i\left(\tilde{A}(P), \zeta^{\prime \prime}\right)}
$$

is a periodic function of the coordinates $\zeta_{k}^{\prime}, \zeta_{k}^{\prime \prime}$. For real $x, t$ the function $\psi$ can be represented in the form

$$
\tilde{\psi}_{0}=\frac{\phi(\widehat{U} x+\widehat{V} t+\zeta, P)}{\phi(\zeta, P)} e^{t p_{-}+x p+} .
$$

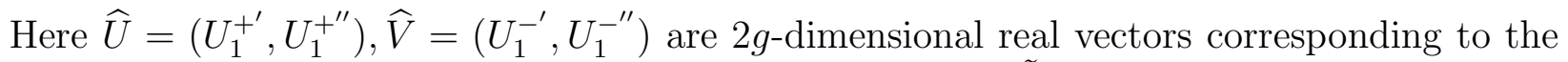
complex vectors $U, V ; d p_{ \pm}$are meromorphic differentials on $\tilde{\Gamma}$ with poles of the second order at $P_{ \pm}$and whose periods are pure imaginary.

The differential $d \tilde{\psi}_{0}$ is also a solution of the same Schrödinger equation. That implies the equality

$$
\partial_{x}\left(\partial_{t} \tilde{\psi}_{0}^{\sigma} d \tilde{\psi}_{0}-\tilde{\psi}_{0}^{\sigma} \partial_{t} d \tilde{\psi}_{0}\right)=\partial_{t}\left(\partial_{x} \tilde{\psi}_{0}^{\sigma} d \tilde{\psi}_{0}-\tilde{\psi}_{0}^{\sigma} \partial_{x} d \tilde{\psi}_{0}\right)
$$

The "averaging" of this equation in the variables $x, t$ gives the equation

$$
\left\langle\partial_{t} \tilde{\psi}_{0}^{\sigma} \tilde{\psi}_{0}-\tilde{\psi}_{0}^{\sigma} \partial_{t} \tilde{\psi}_{0}\right\rangle_{x} d p_{-}=\left\langle\partial_{x} \tilde{\psi}_{0}^{\sigma} \tilde{\psi}_{0}-\tilde{\psi}_{0}^{\sigma} \partial_{x} \tilde{\psi}_{0}\right\rangle_{t} d p_{+} .
$$

Here $\langle\cdot\rangle_{t}$ stands for the mean value in $t$ defined as in (4.51), and $\langle\cdot\rangle_{x}$ stands for the mean value in $x$ defined in a similar way. The same arguments as in [17], show that the differential

$$
d \Omega=\frac{d p_{+}}{\left\langle\partial_{t} \tilde{\psi}_{0}^{\sigma} \tilde{\psi}_{0}-\tilde{\psi}_{0}^{\sigma} \partial_{t} \tilde{\psi}_{0}\right\rangle_{x}}=\frac{d p_{-}}{\left\langle\partial_{x} \tilde{\psi}_{0}^{\sigma} \tilde{\psi}_{0}-\tilde{\psi}_{0}^{\sigma} \partial_{x} \tilde{\psi}_{0}\right\rangle_{t}}
$$

is holomorphic on $\tilde{\Gamma}$ except at the branch points where it has simple poles. It has zeros at the poles of $\tilde{\psi}_{0}$ and $\tilde{\psi}_{0}^{\sigma}$. The lemma is proved.

The differential $\tilde{\psi}_{0} \tilde{\psi}_{0}^{\sigma} d \Omega$ is a meromorphic differential on $\tilde{\Gamma}$. Its residues at the points $P_{ \pm}$ are equal to \pm 1 , respectively. Therefore, sum of its residues at the points $Q_{k}^{i}$ equals zero, i.e.,

$$
\sum_{i, k} \tilde{r}_{k}^{i} \tilde{\psi}_{0}\left(t, Q_{k}^{i}\right) \tilde{\psi}_{0}^{\sigma}\left(t, Q_{k}^{i}\right)=0, \quad \tilde{r}_{k}^{i}=\operatorname{res}_{Q_{k}^{i}} d \Omega .
$$

Note, that equation (4.72) is sufficient for the potential reduction of the Schrödinger operator, which is equivalent to the equation $\tilde{\psi}_{0}\left(t, P_{+}\right)=1$.

From (4.60) it follows that the evaluations of $\tilde{\psi}_{0}$ at the points $Q_{k}^{i}$ satisfy linear equations

$$
\sum_{i=1}^{\nu_{k}} \tilde{c}_{k, j}^{i} \tilde{\psi}_{0}\left(t, Q_{k}^{i}\right)=0, \quad j=1, \ldots, n_{k} .
$$


Note, that the normalization of $\psi_{0}$ at $t=0$ implies

$$
\sum_{i=1}^{\nu_{k}} \tilde{c}_{k, j}^{i}=0
$$

The coefficients $\tilde{c}_{k, j}^{i}$ in (4.73) are unique up to the transformations $\tilde{c}_{k, j}^{i} \rightarrow \sum_{l} g_{k, j}^{l} \tilde{c}_{k, l}^{i}$, where $g_{k}=\left\{g_{k, j}^{l}\right\}$ are $t$-independent non-degenerate matrices. In what follows we normalize $\tilde{c}_{k}$ by the condition

$$
\tilde{c}_{k, j}^{i}=\delta_{j}^{i}, i=1, \ldots, n_{k} .
$$

In that gauge the matrix elements $\tilde{c}_{k, j}^{i}(Z), i>n_{k}$ become well-defined abelian functions on $X$.

Equations (4.72) should follow from equations (4.73). The evaluations of $\tilde{\psi}_{0}$ at the preimages of any two distinct singular points $Q_{k}, Q_{k}^{\prime}, k \neq k^{\prime}$ are independent. That implies the following orthogonality relations

$$
\sum_{j=1}^{n_{k}} \tilde{r}_{k}^{j} \tilde{c}_{k, j}^{i} \tilde{c}_{k, j}^{i}=-\tilde{r}_{k}^{i}, \quad \sum_{j=1}^{n_{k}} \tilde{r}_{k}^{j} \tilde{c}_{k, j}^{i} \tilde{c}_{k, j}^{i^{\prime}}=0, \quad n_{k}<i \neq i^{\prime} \leq \nu_{k} .
$$

(Compare (4.76) with the orthogonality conditions established in $([5]))$.

Corollary 4.5 The multiplicity $\nu_{k}$ of the singular point $Q_{k}$ of the spectral curve is equal to $\nu_{k}=2 n_{k}$, where $n_{k}$ is the number of the linear relations in (4.73).

Proof. As it was shown above, for the generic $Z$ the $\operatorname{ring} \mathcal{A}^{Z}$ is maximal. The ring $\mathcal{A}^{Z}$ is maximal if and only if for each $k$ the linear subspace $W_{k} \subset \mathbb{C}^{2 \nu_{k}}$ defined by the equations $\sum_{i} \tilde{c}_{k, j}^{i}(Z) w_{k}^{i}=0$ is invariant under the multiplication by a diagonal matrix $H_{k}$ only if $H_{k}$ is a scalar matrix. The last condition implies that each $\left(n_{k} \times n_{k}\right)$ minor of the matrix $c_{k}=\left\{\tilde{c}_{k, j}^{i}\right\}$ with $n_{k}<i \leq \nu_{k}$ is non-degenerate. The columns of the matrix $\tilde{c}_{k, j}^{i}$ with $i>n_{k}$ are "orthogonal" to each other. Then, non-degeneracy of all the corresponding minors implies that the number $\nu_{k}-n_{k}$ of such columns is not bigger than the dimension $n_{k}$ of the column vectors, i.e. $\nu_{k} \leq 2 n_{k}$.

From (4.66) it follows that the degree of the pole divisor $D$ equals $\operatorname{deg} D=\tilde{g}+1 / 2 \sum_{k} \nu_{k}$, where $\tilde{g}$ is the genus of $\tilde{\Gamma}$. The uniqueness of the function $\psi_{0}$ defined by $D$ and the relations (4.73), imply that $\operatorname{deg} D=\tilde{g}+\sum_{k} n_{k}$. The latter equations imply $\sum_{k}\left(2 n_{k}-\nu_{k}\right)=0$. As shown above, each term of the sum is non-negative. Hence, $\nu_{k}=2 n_{k}$ and the corollary is thus proven.

Lemma 4.12 There exist constants $r_{k}^{i}$ such that the equation

$$
\sum_{i=1}^{2 n_{k}} r_{k}^{i} \theta\left(A_{k}^{i}+Z\right) \theta\left(A_{k}^{i}-Z\right)=0, \quad A_{k}^{i}=\tilde{A}\left(Q_{k}^{i}\right)
$$

holds. 
Proof. Taking the square of (4.74) and using (4.76) we get the equation

$$
\sum_{i=1}^{2 n_{k}} \tilde{r}_{k}^{i}=0
$$

The residues of the differential $d \Omega$ are well-defined abelian functions $\tilde{r}_{k}^{i}(Z)$ on $X$. The pole divisor of all the residues coincides with the zero divisor of $\theta$. The residue of $d \Omega$ at $Q_{k}^{i}$ equals zero, when the pole divisor of $\tilde{\psi}_{0}$ contains the puncture $P_{-}$. Therefore, from (4.65) it follows that $\tilde{r}_{k}^{i}$ has the form

$$
\tilde{r}_{k}^{i}(Z)=r_{k}^{i} \frac{\theta\left(A_{k}^{i}+Z\right) \theta\left(A_{k}^{i}-Z\right)}{\theta^{2}(Z)},
$$

where $r_{k}^{i}$ are constants. Equations (4.78) and (4.79) imply (4.77) and the lemma is proved.

Our next and the final goal is to show that $n_{k}=1$, i.e. all of the singular points of $\Gamma$ are simple double points, as it is stated in the main theorem.

If $n_{k}>1$, then from indecomposability of the matrix $\tilde{c}_{k, j}^{i}(Z)$ at the generic $Z$ it follows that all the points $A_{k}^{i}$ are distinct, $A_{k}^{i} \neq A_{k}^{j}$. That and the formula (4.65) for $\tilde{\psi}_{0}$ imply that in the gauge (4.75) the coefficient $\tilde{c}_{k, j}^{i}$ for $i>n_{k}$ has pole at the divisor $\theta\left(A_{k}^{j}+Z\right)=0$ and zero at the divisor $\theta\left(A_{k}^{i}+Z\right)=0$.

Let us fix a pair of indices $m, l>n_{k}$ and define a set $\mathcal{D}_{k}^{m, l} \subset X$ by the equations:

$$
\tilde{c}_{k, 1}^{i}(Z)=0, \quad n_{k}<i \neq m, l .
$$

On $\mathcal{D}_{k}^{m, l}$ equation (4.74) takes the form

$$
1+\tilde{c}_{k, 1}^{m}(Z)+\tilde{c}_{k, 1}^{l}(Z)=0, \quad Z \in \mathcal{D}_{k}^{m, l} .
$$

From (4.80) and the orthogonality conditions (4.76) it follows that on $\mathcal{D}_{k}^{m, l}$ the equation $\tilde{c}_{k, 1}^{l}(Z)=0$ implies

$$
\tilde{c}_{k, j}^{m}(Z)=0, \quad j=n_{k}+2, \ldots, 2 n_{k} .
$$

Then, from (4.82) it follows that

$$
Z \in \mathcal{D}_{k}^{m, l}, \tilde{c}_{k, 1}^{l}(Z)=0 \Rightarrow \tilde{r}_{k}^{1}+\tilde{r}_{k}^{m}=0 .
$$

Hence, $\tilde{c}_{k, 1}^{l}$, restricted to $\mathcal{D}_{k}^{m, l}$, is of the form

$$
\tilde{c}_{k, 1}^{l}=\frac{\tilde{r}_{k}^{1}+\tilde{r}_{k}^{m}}{h(Z)} \cdot \frac{\theta\left(A_{k}^{l}+Z\right)}{\theta\left(A_{k}^{1}+Z\right)} \cdot \theta^{2}(Z), \quad Z \in \mathcal{D}_{k}^{m, l},
$$

where $h$ is a holomorphic section of the line bundle of $\left|2 \Theta-A_{k}^{l}+A_{k}^{1}\right|$ restricted to $\mathcal{D}_{k}^{m, l}$. (Recall, that $\tilde{c}_{k, j}^{i}$ and $\tilde{r}_{k}^{i}$ are abelian functions.)

The same arguments imply that on $\mathcal{D}_{k}^{m, l}$ zeros of $\tilde{c}_{k, 1}^{m}$ are in the zero divisor of $\tilde{r}_{k}^{1}+\tilde{r}_{k}^{l}$. Then using (4.81) we get

$$
\tilde{c}_{k, 1}^{m}=g \frac{\tilde{r}_{k}^{1}+\tilde{r}_{k}^{l}}{h(Z)} \cdot \frac{\theta\left(A_{k}^{m}+Z\right)}{\theta\left(A_{k}^{1}+Z\right)} \cdot \theta^{2}(Z), \quad Z \in \mathcal{D}_{k}^{m, l},
$$


where $g$ is a constant. Therefore, $h$ is a section of the restriction to $\mathcal{D}_{k}^{m, l}$ of the line bundle $\left|2 \Theta-A_{k}^{m}+A_{k}^{1}\right|$. Hence, $A_{k}^{m}=A_{k}^{l}$. The choice of points $A_{k}^{m}, A_{k}^{l}$ was arbitrary, Therefore, we have proved that all the points $A_{k}^{i}=A_{k}$ do coincide. In that case, equations (4.73) are equivalent to $\left(2 n_{k}-1\right)$ equations $\tilde{\psi}_{0}\left(t, Q_{k}^{i}\right)=\tilde{\psi}_{0}\left(t, Q_{k}^{j}\right)$. That implies $2 n_{k}-1=n_{k}=1$, i.e., $\Gamma$ has only simple double singular points. For such a curve all the sheafs $\mathcal{F}$ are line bundles. Therefore, the map $j$ in (4.35) is inverse to $i_{Z}$ in (4.58) and the main theorem is thus proven.

\section{Appendix.}

Theorem 5.1 (R.Friedman) Let $\Gamma$ be an irreducible projective curve, with an involution $\sigma$, and suppose that the generalized Prym variety $P(\Gamma, \sigma)$ is compact. Then every singular point $x$ of $\Gamma$ is a fixed point of $\sigma$, the singularity at $x$ is locally analytically isomorphic to a union of coordinate axes in a neighborhood of the origin in $\mathbb{C}^{N}$ for some $N$, and in a neighborhood of such a singular point $\sigma$ fixes each of the local analytic branches.

Proof Let $p: \widetilde{\Gamma} \rightarrow \Gamma$ be the normalization of $\Gamma$. The involution $\sigma$ lifts to an involution on $\widetilde{\Gamma}$, also denoted $\sigma$. The sheaf $p_{*}\left(\mathcal{O}_{\widetilde{\Gamma}}\right) / \mathcal{O}_{\Gamma}$ is supported at the finitely many points of $\Gamma_{\text {sing }}$. The cohomology long exact sequence for

$$
0 \rightarrow \mathcal{O}_{\Gamma} \rightarrow p_{*}\left(\mathcal{O}_{\widetilde{\Gamma}}\right) \rightarrow p_{*}\left(\mathcal{O}_{\widetilde{\Gamma}}\right) / \mathcal{O}_{\Gamma} \rightarrow 0
$$

yields a long exact sequence

$$
\begin{aligned}
0 \rightarrow H^{0}\left(\Gamma ; \mathcal{O}_{\Gamma}\right) \rightarrow H^{0}\left(\Gamma ; p_{*}\left(\mathcal{O}_{\widetilde{\Gamma}}\right)\right)=H^{0}\left(\widetilde{\Gamma} ; \mathcal{O}_{\widetilde{\Gamma}}\right) \rightarrow H^{0}\left(\Gamma ; p_{*}\left(\mathcal{O}_{\widetilde{\Gamma}}\right) / \mathcal{O}_{\Gamma}\right) \\
\rightarrow H^{1}\left(\Gamma ; \mathcal{O}_{\Gamma}\right) \rightarrow H^{1}\left(p_{*}\left(\mathcal{O}_{\widetilde{\Gamma}}\right)\right)=H^{1}\left(\widetilde{\Gamma} ; \mathcal{O}_{\widetilde{\Gamma}}\right) \rightarrow 0 .
\end{aligned}
$$

Since $\widetilde{\Gamma}$ is irreducible, $H^{0}\left(\widetilde{\Gamma} ; \mathcal{O}_{\widetilde{\Gamma}}\right)=\mathbb{C}$, so that there is an exact sequence

$$
0 \rightarrow H^{0}\left(\Gamma ; p_{*}\left(\mathcal{O}_{\widetilde{\Gamma}}\right) / \mathcal{O}_{\Gamma}\right) \rightarrow H^{1}\left(\Gamma ; \mathcal{O}_{\Gamma}\right) \rightarrow H^{1}\left(\widetilde{\Gamma} ; \mathcal{O}_{\widetilde{\Gamma}}\right) \rightarrow 0
$$

Here $H^{1}\left(\Gamma ; \mathcal{O}_{\Gamma}\right)$ is the tangent space to the generalized Jacobian of $\Gamma$ and the subspace $H^{0}\left(\Gamma ; p_{*}\left(\mathcal{O}_{\widetilde{\Gamma}}\right) / \mathcal{O}_{\Gamma}\right)$ is the tangent space to its noncompact part. If $V$ is a vector space on which $\sigma$ acts, let $V^{-}$denotes the anti-invariant part of $V$, i.e. the $(-1)$-eigenspace. Then the tangent space $T_{P(\Gamma, \sigma)}$ to $P(\Gamma, \sigma)$ fits into an exact sequence

$$
0 \rightarrow H^{0}\left(\Gamma ; p_{*}\left(\mathcal{O}_{\widetilde{\Gamma}}\right) / \mathcal{O}_{\Gamma}\right)^{-} \rightarrow T_{P(\Gamma, \sigma)} \rightarrow T_{P(\widetilde{\Gamma}, \sigma)} \rightarrow 0
$$

It follows that $P(\Gamma, \sigma)$ is compact if and only if $H^{0}\left(\Gamma ; p_{*}\left(\mathcal{O}_{\widetilde{\Gamma}}\right) / \mathcal{O}_{\Gamma}\right)^{-}=0$.

First let us show that, for all $x \in \Gamma_{\text {sing }}, \sigma(x)=x$. There is an isomorphism

$$
H^{0}\left(\Gamma ; p_{*}\left(\mathcal{O}_{\widetilde{\Gamma}}\right) / \mathcal{O}_{\Gamma}\right)=\bigoplus_{x \in \Gamma_{\text {sing }}} \widetilde{R}_{x} / R_{x}
$$

where $R_{x}$ is the local ring $\mathcal{O}_{\Gamma, x}$ and $\widetilde{R}_{x}$ is its normalization. Clearly, $\sigma$ induces an isomorphism $\widetilde{R}_{x} / R_{x} \cong \widetilde{R}_{\sigma(x)} / R_{\sigma(x)}$. If $\sigma(x) \neq x$, and $\alpha$ is a nonzero element of $\widetilde{R}_{x} / R_{x}$, then $\alpha-\sigma(\alpha) \in$ 
$H^{0}\left(\Gamma ; p_{*}\left(\mathcal{O}_{\widetilde{\Gamma}}\right) / \mathcal{O}_{\Gamma}\right)$ is nonzero, a contradiction. Hence $\sigma(x)=x$. Moreover, for all $\alpha \in \widetilde{R}_{x} / R_{x}$ $\sigma(\alpha)=\alpha$.

We now fix attention on a given $x \in \Gamma_{\text {sing, }}$, and write $R=R_{x}$ and $\widetilde{R}=\widetilde{R}_{x}$. Note that, if $y_{1}, \ldots, y_{n}$ are the preimages of $x$ in $\widetilde{\Gamma}$, and $t_{i}$ is a local analytic coordinate for $\widetilde{\Gamma}$ at $y_{i}$, then $\widetilde{R} \cong \bigoplus_{i} \mathbb{C}\left\{t_{i}\right\}$. Moreover, $R$ is a subalgebra of $\widetilde{R}$, and $\operatorname{dim}_{\mathbb{C}}(\widetilde{R} / R)<\infty$; on particular, $\widetilde{R}$ is a finite $R$-module. Let $\mathfrak{m}_{i}=t_{i} \mathbb{C}\left\{t_{i}\right\}$. Clearly, $R$ is contained in the subalgebra $\mathbb{C} \oplus \bigoplus_{i} \mathfrak{m}_{i}$, and the claim about the analytic nature of the singularities is just the statement that $R=\mathbb{C} \oplus \bigoplus_{i} \mathfrak{m}_{i}$.

Next we claim that $\sigma$ does not permute the analytic branches through $x$. If it did, then the action of $\sigma$ on $\widetilde{R}$ would exchange two factors $\mathbb{C}\left\{t_{i}\right\}$ and $\mathbb{C}\left\{t_{j}\right\}$ for $j \neq i$. In this case, let $e_{i}$ be the image of $1 \in \mathbb{C}\left\{t_{i}\right\}$ in $\widetilde{R}$. Then $\sigma\left(e_{i}\right)-e_{i} \in(\widetilde{R})^{-}$, and $\sigma\left(e_{i}\right)-e_{i} \notin R$. Thus $\sigma\left(e_{i}\right)-e_{i}$ is a nonzero class in $(\widetilde{R} / R)^{-}=(\widetilde{R})^{-} / R^{-}$, a contradiction. Thus, for every $i, \sigma$ fixes $\mathbb{C}\left\{t_{i}\right\}$ and induces a holomorphic involution on the corresponding branch of $\widetilde{\Gamma}$.

We can thus choose the coordinate $t_{i}$ so that $\sigma\left(t_{i}\right)=-t_{i}$. Since $(\widetilde{R} / R)^{-}=(\widetilde{R})^{-} / R^{-}=0$, $t_{i} \in R$ for every $i$. Clearly, $t_{i} \in \mathfrak{m}$, where $\mathfrak{m}$ is the maximal ideal of the local ring $R$. Now let $\widehat{R}=\mathbb{C} \oplus \bigoplus_{i} \mathfrak{m}_{i} \subseteq \widetilde{R}$. As noted above, $R \subseteq \widehat{R}$ and we must show that $R=\widehat{R}$. In any case, $\widehat{R}$ is a finite $R$-module. Given $r \in \widehat{R}$, there exists a $c \in \mathbb{C} \subseteq R \subseteq \widehat{R}$ such that $r-c \in \bigoplus_{i} \mathfrak{m}_{i}=\left(t_{1}, \ldots, t_{n}\right) \widehat{R} \subseteq \mathfrak{m} \widehat{R}$. Thus $\widehat{R}=R+\mathfrak{m} \widehat{R}$, and so by Nakayama's lemma $R=\widehat{R}$.

Acknowledgments. The author wishes to thank Sam Grushevski for very useful conversation on the subject of this paper, Robert Friedman for the communication of the proof of Corollary 4.4. The author is grateful to Takahiro Shiota whose remarks helped the author to clarify some missing arguments in the first version of the paper.

\section{References}

[1] I.Taimanov, Secant of abelian varieties, theta-functions and soliton equations, Russian Math. Surveys, 52 (1997), n. 1, 149-224.

[2] V.Shokurov, Prym varieties: theory and applications, Izvestiya AN SSSR, ser. matem. 47 (1984), n.4, 785-856.

[3] T. Shiota, Characterization of Jacobian varieties in terms of soliton equations, Invent. Math., 83(2):333-382, 1986.

[4] I.Taimanov, Prym varieties of branch covers and npnlinear equations, Matem. Sbornik, 181(1990), n 7, 934-950.

[5] T.Shiota, Prym varieties and soliton equations, in: Infinite-dimensional Lie algebras and groups (Luminy-Marseille, 1988), Adv.ser.Math.Phys., 7 Teaneck: World Sci.Publishing, 1989, 407-448. 
[6] A. Beauville, O. Debarre, Sur le probleme de Schottky pour les varieites de Prym, (French) [On the Schottky problem for Prym varietes] Ann. Scuola Norm. Sup. Pisa Cl. Sci. (4) 14 (1987), no. 4, 613-623 (1988).

[7] I.Krichever, Integrable linear equations and the Riemann-Schottky problem, math. $\mathrm{AG} / 0504192$

[8] E. Arbarello, I. Krichever, G. Marini, Characterizing Jacobians via flexes of the Kummer Variety, Math. Res. Lett. 13 (2006), no. 1, 109-123.

[9] G. E. Welters, A criterion for Jacobi varieties, Ann. of Math., 120 (1984), n. 3, 497-504.

[10] S. Novikov, A. Veselov, Finite-gap two-dimensional periodic Schrödinger operators: exact formulae and evolution equations, Dokladi AN USSR, 279, 1984, n 1, 20-24.

[11] I. Krichever and D.H. Phong, Symplectic forms in the theory of solitons, Surveys in Differential Geometry IV (1998), edited by C.L. Terng and K. Uhlenbeck, 239-313, International Press, hep-th/9708170

[12] I. M. Krichever, Integration of non-linear equations by methods of algebraic geometry, Funct. Anal. Appl., 11 (1977), n. 1, 12-26.

[13] I. M. Krichever, Methods of algebraic geometry in the theory of non-linear equations, Russian Math. Surveys, 32 (1977), n. 6, 185-213.

[14] J.L. Burchnall, T.W. Chaundy, Commutative ordinary differential operators.I, Proc. London Math Soc. 21 (1922), 420-440.

[15] J.L. Burchnall, T.W. Chaundy, Commutative ordinary differential operators.II, Proc. Royal Soc.London 118 (1928), 557-583.

[16] B.A.Dubrovin, I.M.Krichever, S.P.Novikov Schrödinger equation in magnetic field and Riemann surfaces, Doklady AN USSR 229 (1976), n 1, 15-18.

[17] I.Krichever, Spectral theory of two-dimensional periodic operators and its applications, Uspekhi Mat. Nauk 44 (1989), n 2, 121-184.

[18] I.Taimanov, Funct. Anal. Appl., 24(1990), n.1, 86-87.

[19] E.Arbarello and C. De Concini, Another proof of a conjecture of S.P. Novikov on periods of abelian integrals on Riemann surfaces, Duke Math. Journal, 54 (1987), 163-178.

[20] I.Krichever, S.Novikov Holomorphic bundles over algebraic curves and non-linear equations, Uspekhi Mat. Nauk 35 (1980), n 6, 47-68.

[21] D. Mumford, An algebro-geometric construction of commuting operators and of solutions to the Toda lattice equation, Korteweg-de Vries equation and related non-linear equations - Proceedings Int.Symp. Algebraic Geometry, Kyoto, 1977, 115-153, Kinokuniya Book Store, Kyoto, 1978. 
[22] G.Segal, G.Wilson, Loop groups and equations of KdV type, IHES Publ.Math. 61, 1985, 5-65.

[23] I.Krichever, Averaging method for two-dimentional integrable equations, Funct. Anal. Appl. 22 (1988), no. 3, 200-213.

[24] P. Deligne, D. Mumford, The irreducibility of the space of curves of given genus. Inst. Hautes tudes Sci. Publ. Math. No. 361969 75-109 